\title{
Transport through open quantum dots: Making semiclassics quantitative
}

\author{
Iva Březinová, ${ }^{1, *}$ Ludger Wirtz, ${ }^{2}$ Stefan Rotter, ${ }^{1}$ Christoph Stampfer, ${ }^{3}$ and Joachim Burgdörfer ${ }^{1}$ \\ ${ }^{1}$ Institute for Theoretical Physics, Vienna University of Technology, Wiedner Hauptstraße 8-10/136, 1040 Vienna, Austria, EU \\ ${ }^{2}$ Department ISEN, Institute for Electronics, Microelectronics, and Nanotechnology (IEMN), CNRS-UMR 8520, B.P. 60069, \\ 59652 Villeneuve d'Ascq Cedex, France, EU \\ ${ }^{3}$ JARA-FIT and II. Institute of Physics, RWTH Aachen, 52074 Aachen, Germany, EU
}

(Received 28 October 2009; published 9 March 2010)

\begin{abstract}
We investigate electron transport through clean open quantum dots (quantum billiards). We present a semiclassical theory that allows to accurately reproduce quantum-transport calculations. Quantitative agreement is reached for individual energy-dependent and magnetic field dependent elements of the scattering matrix. Two key ingredients are essential: (i) inclusion of pseudopaths which have the topology of linked classical paths resulting from diffraction in addition to classical paths and (ii) a high-level approximation to diffractive scattering. Within this framework of the pseudopath semiclassical approximation (PSCA), typical shortcomings of semiclassical theories such as violation of the anticorrelation between reflection and transmission and the overestimation of conductance fluctuations are overcome. Beyond its predictive capabilities the PSCA provides deeper insights into the quantum-to-classical crossover.
\end{abstract}

DOI: 10.1103/PhysRevB.81.125308 PACS number(s): 73.23.-b, 73.63.Kv, 05.45.Mt, 72.15.Rn

\section{INTRODUCTION}

The ability to controllably fabricate, manipulate, and examine structures on the submicrometer scale has let to the observation of quantum phenomena in electron transport such as, e.g., universal conductance fluctuations in chaotic billiards and weak localization (WL), which dominate transport at the nanoscale. ${ }^{1,2}$ By reducing the characteristic system size below the electronic inelastic mean free path, transport enters the so-called ballistic regime. ${ }^{3}$ Ballistic electron transport is a prime candidate for semiclassical descriptions ${ }^{4-26}$ where the classical trajectories carry an amplitude which reflects the stability of the classical orbits and a phase which contains the classical action and accounts for quantum interference. ${ }^{27}$ On a more fundamental level, the semiclassical framework provides a conceptually powerful bridge between classical and quantum mechanics allowing an intuitive approach to quantum mechanics and quantum chaos, in general, and to transport through open quantum dots or so-called quantum billiards, in particular. ${ }^{4-6}$

Several semiclassical approximations (SCAs) based on the approximation of the constant-energy Green's function for propagation in a billiard have been proposed and compared with numerical quantum-transport calculations or experiment. ${ }^{8-19,28,29}$ While many qualitative features could be well reproduced, quantitative agreement on a systemspecific level has remained a challenge.

One underlying difficulty is the multiscale nature of the quantum-to-classical transition for transport through open quantum dots. For the semiclassical approximation to hold, the de Broglie wavelength $\lambda$ should be vanishingly small compared to all characteristic dimensions of the device. Such asymptotic theories have been successfully employed to reproduce, upon ensemble averaging, random matrix theory results for chaotic cavities (see, e.g., Refs. 7 and 20-23). A quantitative comparison on a system-specific level with full quantum calculations or experiments is, however, only possible in the nonasymptotic regime where $\lambda$ is small compared to the linear dimension $D$ of the dot, $\lambda \ll D$ but still comparable to the width of the lead (or quantum wire) $d, \lambda \lesssim d$. Moreover, for billiards with sharp edges the proper asymptotic limit is, rigorously, out of reach. The present theory addresses this nonasymptotic semiclassical regime, often also referred to as the "near" semiclassical regime. We show that the proper inclusion of diffractive contributions allows to quantitatively reproduce quantum calculations. The diffractive coupling between classical paths gives rise to pseudopaths that are missing in the standard SCA and are the key to remedy many of the deficiencies of semiclassical approximations.

We show in the present communication that this pseudopath semiclassical approximation (PSCA) can reach quantitative agreement with full quantum simulations provided a high-order diffraction theory for the coupling between classical paths is used. For the scattering at the leads we develop an approximation involving elements of both the uniform theory of diffraction (UTD) (Refs. 30 and 31) and the geometric theory of diffraction (GTD) (Ref. 32) referred to in the following as the GTD-UTD approximation. With these ingredients good agreement with quantum simulations is found.

One key conceptual insight is the unambiguous identification of the paths that contribute to quantum transport. We apply the present theory to a circle-shaped regular quantum dot for which the enumeration of paths, more precisely of path bundles, is easily possible. Unlike for chaotic dots, for which the exponential proliferation of contributing paths as a function of the path length makes their unique identification difficult, for regular systems their enumeration and identification is straightforward up to large path lengths. ${ }^{33}$

The circular billiard is depicted in Fig. 1. The leads are attached at right angle and have equal width $d$. In order to probe the local topology of the cavity we require sufficiently long dwell times such that $d / \rho \ll 1$. The wavelength of the electron $\lambda$ fulfills $\lambda \ll \rho$ for the semiclassical limit to hold inside the cavity. However, as in experimental or numerical studies of quantum billiards $\lambda \lesssim d$. Our semiclassical theory 


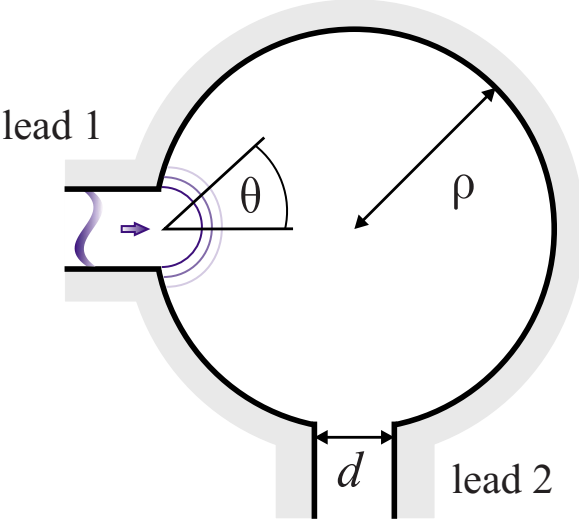

FIG. 1. (Color online) Geometry of the circular billiard of radius $\rho$ with perpendicular leads of equal width $d$. In accordance with previous work (Refs. 34-36) on the circular and the stadium billiard of equal area $a=4+\pi$, we choose $d=0.25$ and $\rho=\sqrt{1+4 / \pi}$.

can thus be quantitatively compared with quantummechanical numerical calculations for the circular billiard.

This paper is organized as following: In Sec. II, we review both the standard SCA as well as the PSCA. These approximations differ by the different path sets entering the corresponding Green's function. In Sec. III, we introduce the GTD-UTD diffraction approximation which is a key to the quantitative agreement between the PSCA and quantum mechanics for transport properties. The important role of pseudopaths and a proper diffraction theory is demonstrated on the level of quantum-mechanical length-area spectra ${ }^{16}$ in Sec. IV. Finally, we compare in Sec. V the semiclassical predictions for a variety of quantum-transport properties that play a key role in the understanding of the quantum-toclassical crossover, in particular, conductance fluctuations (CF), WL, and quantum (nonthermal) shot noise, with quantum calculations.

\section{QUANTUM TRANSPORT THROUGH BILLIARDS}

We consider ballistic transport through quantum billiards for which transport properties are determined by the wave number $(k)$ and magnetic field $(B)$ dependent quantummechanical Hamiltonian $S$-matrix. Dissipative or dephasing processes are neglected. (We will return to the effect of decoherence below.) Moreover, we refer to a "clean" billiard when elastic scattering at a disorder potential in the interior of the structure is absent. In this limit, the $S$-matrix represents elastic scattering at the boundaries of the billiard only.

The $S$-matrix elements $S_{n, m}^{(j, i)}(k, B)$ describe the scattering from transverse mode $m$ in lead $i$ to mode $n$ in lead $j$. We denote the transmission amplitudes from lead 1 to lead 2 as $t_{n m}(k, B)=S_{n m}^{(2,1)}(k, B)$ and the reflection amplitudes back into lead 1 as $r_{n m}(k, B)=S_{n m}^{(1,1)}(k, B)$. According to the Landauer formula the conductance $g$ of a quantum billiard is directly proportional to the total transmission $T(k, B)$,

$$
g(k, B)=\frac{2 e^{2}}{h} T(k, B)=\frac{2 e^{2}}{h} \sum_{n=1}^{M} \sum_{m=1}^{M}\left|t_{n m}(k, B)\right|^{2},
$$

where $M$ is the number of open modes in the leads having equal width $d$. The $S$-matrix elements can be determined by a projection of the lead modes $\varphi_{m}\left(y_{i}\right)\left(y_{i}\right.$ is the transverse coordinate in the lead) onto the Green's function $G\left(y_{j}, y_{i}, k, B\right)$ for propagation from $y_{i}$ to $y_{j}$ at the cavity-lead junction of lead $i$ and lead $j$, respectively. The $S$-matrix elements at $B=0$ are given by the Fisher-Lee equations ${ }^{37}$

$$
\begin{aligned}
t_{n m}(k, B=0) & =-i \sqrt{k_{x_{2}, n} k_{x_{1}, m}} \\
& \times \int d y_{2} \int d y_{1} \varphi_{n}^{*}\left(y_{2}\right) G\left(y_{2}, y_{1}, k, B=0\right) \varphi_{m}\left(y_{1}\right),
\end{aligned}
$$

where $k_{x_{1}, m}\left(k_{x_{2}, n}\right)$ is the longitudinal wave number in lead 1 (lead 2). The prefactor $\sqrt{k_{x_{2}, n} k_{x_{1}, m}}$ is due to flux normalization. We use atomic units $\left(\hbar=|e|=m_{\text {eff }}=1\right)$. At zero magnetic field, the mode wave functions take the form

$$
\varphi_{m}(y)=\sqrt{\frac{2}{d}} \sin \frac{m \pi y}{d} .
$$

For nonzero magnetic field $B \neq 0$, Eqs. (2) and (3) have to be modified (see, e.g., Refs. 34 and 35, and references therein). We use the modular recursive Green's-function method to calculate the exact quantum-mechanical $S$-matrix elements for a given $k$ and $B$ (for details see Refs. 34 and 35).

\section{A. Semiclassical approximations to transport}

The starting point of semiclassical approximations are the $S$-matrix elements [Eq. (2)]. In a first step, the quantummechanical Green's function $G$ is replaced by a corresponding semiclassical expression which represents the FourierLaplace transform in stationary phase approximation of the semiclassical limit of the Feynman propagator. It describes propagation along classical paths with fixed energy. Depending on the class of paths included, a hierarchy of different semiclassical approximations to the Green's function results. These are to be distinguished from the different level of additional approximations employed in the evaluation of the integral [Eq. (2)] which projects the Green's function onto the lead function. The latter gives rise to another set of semiclassical approximations to the $S$-matrix elements.

We focus first on the replacement of $G$ by a semiclassical approximation. For the latter we consider two different levels of approximation, the standard SCA and the PSCA. Both result from the stationary phase approximation (SPA) to the full Feynman propagator reducing the continuous set of paths entering Feynman's path integral to a discrete subset of paths. Assuming that the classical action $S$ is much larger than $\hbar$ and that well localized and separated stationary points with $\delta S_{i}=0$ exist, the standard SCA contains exclusively classical paths. However, near sharp edges of the cavity or near the cavity-lead junctions, the de Broglie wavelength is not negligibly small and the SPA will fail. This leads to diffractive corrections which can be taken into account within the framework of the PSCA. One of its salient features is that the basic notion of a propagator consisting of a sum over a discrete set of paths is preserved. Diffraction effects simply appear as additional contributions to the path sum [see Sec. II C]. 
The classical action for an electron moving along a path $q$ is given by $S_{q}=k L_{q}+B a_{q} / c$, where $L_{q}$ is the length and $a_{q}$ is the directed enclosed area of the path. The enclosed area of open paths is determined according to Ref. 13. $a_{q}$ can have both positive and negative values depending on the rotational direction of the path. In all our semiclassical calculations (standard SCA as well as the PSCA), the magnetic field enters only via the Aharonov-Bohm phase $B a_{q} / c$. The curvature of the paths as well as the effect of nonzero magnetic field on the diffraction at the lead (introduced in Sec. III) is neglected since we consider the regime of weak magnetic fields $(\rho \ll c k / B$ with $\rho$ being the radius of the circular cavity).

\section{B. Standard semiclassical approximation}

The Green's function within the standard SCA entering Eq. (2) is given by ${ }^{4}$

$$
\begin{aligned}
G^{\mathrm{SCA}}\left(y_{j}, y_{i}, k, B\right)= & \frac{2 \pi}{(2 \pi i)^{3 / 2}} \sum_{q: y_{i} \rightarrow y_{j}} \sqrt{\left|D_{q}\left(y_{j}, y_{i}, k\right)\right|} \\
& \times \exp \left[i S_{q}\left(y_{j}, y_{i}, k, B\right)-i \frac{\pi}{2} \mu_{q}\right],
\end{aligned}
$$

where $D_{q}\left(y_{j}, y_{i}, k\right)$ is the deflection factor (a measure for the divergence of nearby trajectories which is inversely proportional to the element $\widetilde{M}_{12}$ of the stability matrix) and $\mu_{q}$ is the Maslov index. The deflection factor is defined as

$$
\left|D_{q}\left(y_{j}, y_{i}, k\right)\right|=\frac{1}{\left|k_{x_{j}}\right|\left|k_{x_{i}}\right|}\left|\frac{\partial^{2} S_{q}}{\partial y_{j} \partial y_{i}}\right|,
$$

where $x_{i}\left(y_{i}\right)$ is the longitudinal (transverse) component of the trajectory's starting point $(i)$ and end point $(j)$, respectively. The Maslov index increases by two for every reflection at the hard-wall boundary of the billiard and by one when passing a focal point along the trajectory. Equation (4) contains a sum over all classical paths $q$ connecting the entrance lead $i$ with the exit lead $j$ (see Sec. II C).

Evaluation of Eq. (2) with $G^{\mathrm{SCA}}$,

$$
\begin{aligned}
t_{n m}^{\mathrm{SCA}}(k, B)= & -i \sqrt{k_{x_{2}, n} k_{x_{1}, m}} \\
& \times \int d y_{2} \int d y_{1} \varphi_{n}^{*}\left(y_{2}\right) G^{\mathrm{SCA}}\left(y_{2}, y_{1}, k, B\right) \varphi_{m}\left(y_{1}\right),
\end{aligned}
$$

proceeds either numerically ${ }^{11}$ or analytically by invoking another set of SPAs. It was recognized from very early on that the SPA as applied to Eq. (6) is poorly justified in the nonasymptotic regime when $\lambda \leq d$. Therefore, various diffraction integral approximations have been proposed..$^{10,12}$ The transmission amplitudes then take the form

$$
\begin{aligned}
t_{n m}^{\mathrm{SCA}}(k, B)= & \frac{-1}{\sqrt{2 \pi i}} \sqrt{k_{x_{2}, n} k_{x_{1}, m}} \sum_{q: y_{i}^{0} \rightarrow y_{j}^{0}} c_{n}\left(\theta_{2}, k, d\right) \\
& \times \sqrt{\left|D_{q}(k)\right|} \exp \left[i S_{q}(k, B)-i \frac{\pi}{2} \mu_{q}\right] c_{m}\left(\theta_{1}, k, d\right),
\end{aligned}
$$

expressed in terms of diffraction coefficients $c_{m}(\theta, k, d)$ describing the diffractive coupling from the entrance lead mode $m$ into the dot and from the dot into the exit lead mode $n$. For path $q$, the angle $\theta_{1}$ stands for the entrance angle into the cavity and $\theta_{2}$ is the exit angle out of the cavity. Deviating from previous calculations, we introduce for $c_{m, n}(\theta, k, d)$ a combination of Keller's GTD (Ref. 32) and the UTD. ${ }^{30,31}$ The derivation of $c_{m}(\theta, k, d)$ within the GTD-UTD is given in Section 2 of the Appendix. The inclusion of diffraction effects in terms of diffraction coefficients in Eq. (7) preserves the structure of the semiclassical transmission amplitude in terms of a discrete sum over paths contributing to transport. The diffraction coefficients provide $\theta$ - and $k$-dependent weighting factors for each path contributing to the transmission from mode $m$ to mode $n$. Within the framework of the diffractive couplings into and out of the leads, the entrance and exit leads are treated as point scatterers. ${ }^{15}$ The point scatterer placed at the center of each cavity-lead junction is marked by $y_{i}^{0}$ in Eq. (7). Each path bundle which connects the entrance and the exit lead is replaced by an appropriately weighted representative path $q$ connecting the center of the entrance lead with the center of the exit lead. Consequently one can replace the deflection factor as given in Eq. (5) by its value in the closed circular billiard $D_{q}$ $=1 / \sqrt{k L_{q}}$, where $L_{q}$ is the length of the path.

The diffractive lead-dot couplings in Eq. (7) should be distinguished from diffractive corrections included in the propagation in the interior of the billiard. We refer to Eq. (7) as the standard SCA while inclusion of diffractive corrections in the billiard corresponds to the PSCA.

\section{Paths entering the standard semiclassical Green's function}

Classical paths in a regular billiard (such as the circle) feature a highly ordered structure of their length and enclosed area distribution (see Fig. 2). The branch structure of the "length-area" distribution of paths connecting the entrance with the exit point is a specialty of the circular cavity. Along each branch the number of bounces off the wall increases by one from one path to its next-higher neighbor. The points of convergence of each branch mark those paths that bounce off the wall infinitely many times and thus run exactly along the cavity boundary. In the limit $\theta \rightarrow \pi / 2$ (where $\theta$ is the entrance angle as given in Fig. 1) each branch contains an infinite number of paths. In our numerical calculations (see Sec. V) paths near this cluster point effectively do not contribute as they are cut off by vanishing diffraction coefficients $c_{n}(\theta \rightarrow \pi / 2, k) \rightarrow 0$.

The distribution of paths eventually reflected back to the entrance point [Fig. 2(a)] is symmetrically distributed relative to the $a=0$ axis due to time-reversal symmetry. Every path has a counterpart of equal length but opposite sign of 

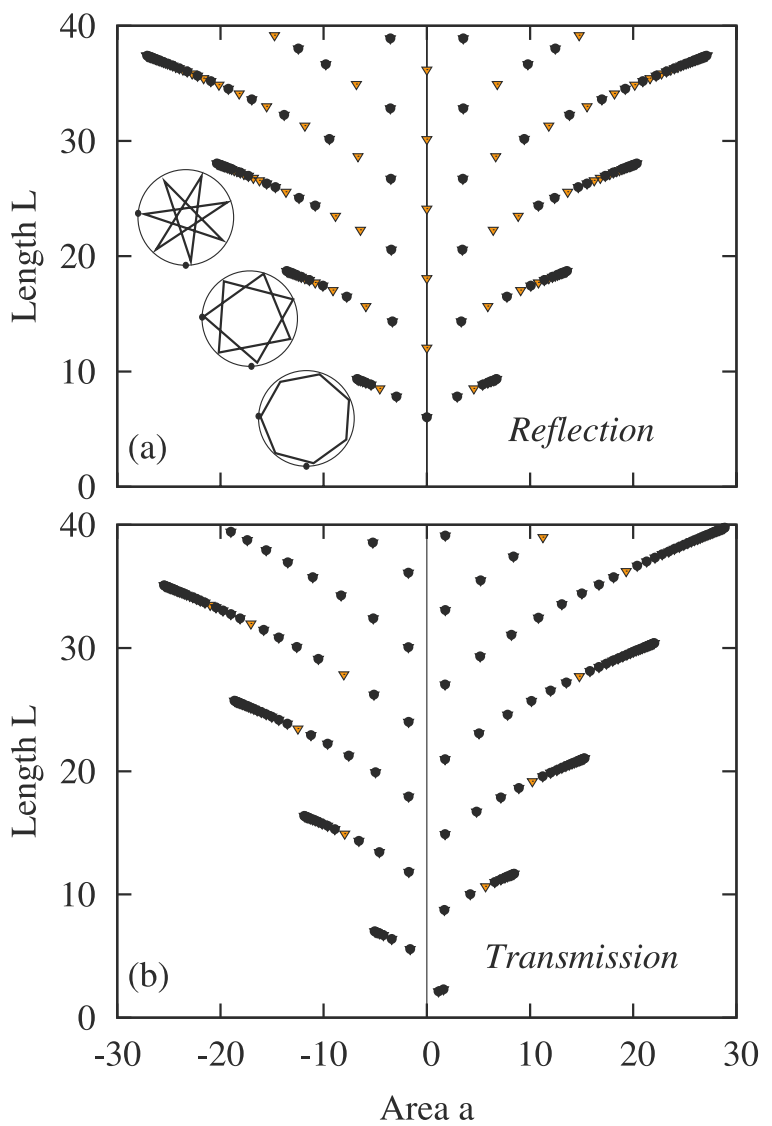

FIG. 2. (Color online) Two-dimensional length vs enclosed area distribution of classical paths within the open (black dots) and closed (orange triangles) circular billiard for (a) reflection and (b) transmission. Each point represents one classical path which connects the centers of each lead. The inset of (a) shows typical paths for the path topology of the three first branches.

the enclosed area. The lowest left and right branches consist of polygons with a number of revolutions $n_{R}=1$ in the cavity. The polygons can be characterized by an angle $\phi=\frac{2 \pi}{n_{C}}$, where $n_{C}$ is the number of corners. Along each branch $n_{C}$ increases by one from one path to the next. The paths of the nexthigher branches revolve twice $\left(n_{R}=2\right)$ around the circle and $\phi=\frac{2 \pi n_{R}}{n_{C}}=\frac{4 \pi}{n_{C}}$. All higher branches can be described analogously.

The branches of transmitted paths are not symmetric relative to the $a=0$ axis but show a clear off-set [Fig. 2(b)]. Path pairs with similar length do not have, in general, the same topology but differ in the number of bounces off the hard wall boundary. As a consequence, these path pairs have different Maslov indices and thus do not interfere constructively. Figure 2 also illustrates the difference between the open and closed billiard, i.e., the effect of "path shadowing" or suppression of longer paths due to their prior exit from the structure. All paths that would be geometrically reflected off the closed lead in the closed system are missing in the open billiard. The difference between the closed and open billiard is particularly evident in reflection since all paths with fourfold symmetry leave the cavity via lead 2 before being reflected back to lead 1 . This is system specific for the circular billiard with perpendicular leads (see Fig. 1).
The distinctly different path distributions for transmission and reflection point already to a clear structural deficiency of the SCA. Many quantum properties of transport are a consequence of the intrinsic coupling between transmission and reflection. The standard SCA does not incorporate this quantum aspect of nonlocality. Classically, the path sets of transmission and reflection are disjoint. The distribution of transmitted paths is markedly different from the one of reflected paths. In quantum transport, transmitted and reflected paths are intertwined and must share the information on the relative phases. A semiclassical theory that reproduces quantum features must therefore allow for coupling between the path sets associated with transmission and reflection. This is the key property of pseudopaths discussed in the following.

\section{Pseudopath semiclassical approximation}

The pseudopath semiclassical approximation ${ }^{14,15}$ goes beyond the standard SCA by systematically including diffractive corrections into the propagation in the interior of the billiard. In the present case, diffractive corrections arise from multiple backscattering, i.e., internal reflections at the leads. We point to the conceptual similarity to "pseudo-orbits" 38 as well as to "diffractive orbits" $31,39,40$ introduced for the study of the density of states in closed billiards and to "Hikami boxes"2,41 introduced for elastic scattering at short-ranged potentials in the interior of a diffusive quantum dot.

In line with multiple-scattering theory the pseudopath semiclassical Green's function can be expressed in terms of a (semiclassical) Dyson equation, ${ }^{15}$

$$
G^{\mathrm{PSCA}}=G^{\mathrm{SCA}}+G^{\mathrm{SCA}} V G^{\mathrm{PSCA}}=G^{\mathrm{SCA}} \sum_{i=0}^{\infty}\left(V G^{\mathrm{SCA}}\right)^{i} .
$$

In the present case, $G^{\mathrm{SCA}}$ plays the role of the unperturbed Green's function and the perturbation "potential" $V$ accounts for the (internal) diffractive scatterings at the lead opening. The unperturbed propagation inside the cavity $G^{\mathrm{SCA}}$ is equivalent to the free propagation in the "closed" system.

For a two-terminal system, the perturbation potential $V$ is given by

$$
V=\left(\begin{array}{cc}
V_{1} & 0 \\
0 & V_{2}
\end{array}\right)
$$

where $V_{1}$ and $V_{2}$ describe the diffractive scattering off lead 1 and lead 2, respectively. The semiclassical Dyson equation, Eq. (8), reads

$$
\begin{aligned}
\left(\begin{array}{ll}
G_{1,1}^{\mathrm{PSCA}} & G_{1,2}^{\mathrm{PSCA}} \\
G_{2,1}^{\mathrm{PSCA}} & G_{2,2}^{\mathrm{PSCA}}
\end{array}\right)= & \left(\begin{array}{ll}
G_{1,1}^{\mathrm{SCA}} & G_{1,2}^{\mathrm{SCA}} \\
G_{2,1}^{\mathrm{SCA}} & G_{2,2}^{\mathrm{SCA}}
\end{array}\right)+\left(\begin{array}{cc}
G_{1,1}^{\mathrm{SCA}} & G_{1,2}^{\mathrm{SCA}} \\
G_{2,1}^{\mathrm{SCA}} & G_{2,2}^{\mathrm{SCA}}
\end{array}\right) \\
& \times\left(\begin{array}{cc}
V_{1} & 0 \\
0 & V_{2}
\end{array}\right)\left(\begin{array}{ll}
G_{1,1}^{\mathrm{PSCA}} & G_{1,2}^{\mathrm{PSCA}} \\
G_{2,1}^{\mathrm{PSCA}} & G_{2,2}^{\mathrm{PSCA}}
\end{array}\right) .
\end{aligned}
$$

To first order, the PSCA to the Green's function (denoted by $\left.G^{\mathrm{PSCA}(1)}\right)$ connecting lead $i$ with lead $j$ includes terms in $V$ of the form, 


$$
\begin{aligned}
G^{\mathrm{PSCA}(1)} & =\sum_{l=1,2} G_{j, l}^{\mathrm{SCA}} V_{l} G_{l, i}^{\mathrm{SCA}} \\
& =\sum_{l=1,2} \sum_{q_{j, l}^{\prime}} \sum_{q_{l, i}} G_{q_{j, l}^{\prime}}^{\mathrm{SCA}} v\left(\theta_{q_{l}^{\prime}}, \theta_{q_{l}}, k, d\right) G_{q_{l, i}}^{\mathrm{SCA}} .
\end{aligned}
$$

Equation (11) may serve as example to illustrate the physics entering Eq. (10). It describes propagation from lead $i$ to lead $j$ via one intermediate visit to lead $l$ where diffractive internal backscattering with amplitude $v\left(\theta_{q_{l}^{\prime}}, \theta_{q_{l}}, k, d\right)$ takes place. Diffractive internal backscattering refers to the reflected component of the wave approaching the lead opening from the inside. The path from lead $i$ to lead $l, q_{l, i}$ as well as from lead $l$ to lead $j, q_{j, l}^{\prime}$, are classical paths described by $G^{\mathrm{SCA}}$. Diffractive scattering couples the incident path $q_{l, i}$ with angle $\theta_{q_{l}}$ to the exiting path $q_{j, l}^{\prime}$ with angle $\theta_{q_{l}^{\prime}}$ thereby coupling two disjoint subsets of classical paths and generating a firstorder pseudopath. The determination of the diffraction coefficient $v\left(\theta_{q_{l}^{\prime}}, \theta_{q_{l}}, k, d\right)$ will be discussed in more detail in Sec. III. From a conceptual point of view, the pseudopath semiclassical approximation [Eq. (10)] is closely related to the diagrammatic perturbation theory. ${ }^{1}$ Both leads 1 and 2 (i.e., $V_{1}$ and $V_{2}$ ) act much like Hikami boxes, ${ }^{2,41}$ where electrons cannot be described semiclassically since the characteristic potential length scale (in our case the sharp edges of the leads) is smaller than the electron wavelength $\lambda$. Thus, the wave nature of electrons has to be taken into account as diffraction at the point scatterers allowing classically distinct path sets to mix. This is crucial for the quantum corrections to the transmission and reflection amplitudes and repairs some of the deficiencies of the standard SCA (see Sec. V).

\section{E. Paths entering the pseudopath semiclassical Green's function}

Within the PSCA pseudopaths are formed by joining classical paths together via diffraction. With each increasing order of the PSCA the length-area distribution gets more and more densely filled with paths, or equivalently, the number of paths increases with the order of the PSCA (Fig. 3). Pseudopaths form product sets of classical paths, e.g., joining a given classical path for transmission with a classical path contributing to reflection (or in reverse order) forms the set of transmitted pseudopaths to first order. Reflected firstorder pseudopaths result from joining two classical transmitted paths or two classical reflected paths. This coupling allows to recover the nonlocality of quantum transport. Higher-order pseudopaths are constructed analogously. With increasing order and increasing (combined) path length the total number of pseudopaths exponentially proliferates. This is in sharp contrast to the power-law growth of purely classical paths for regular systems and explains why the effect of diffractive scattering is more likely visible in regular than in chaotic systems where the exponential proliferation of classical orbits may mask the diffractive contributions.

The length-area distribution of first-order pseudopaths contained in Eq. (10) contributing to reflection is (of course) still symmetric [Fig. 3(a)]. In addition, new branches appear with classical and pseudopath partners of approximately
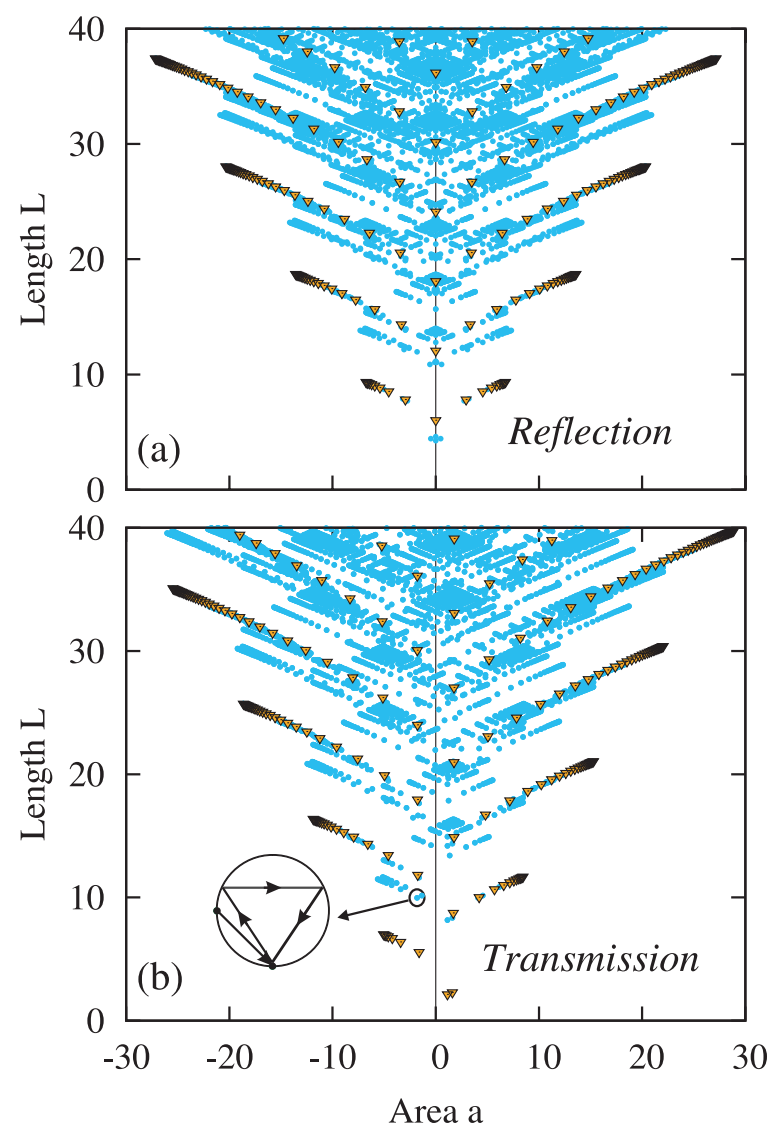

FIG. 3. (Color online) Length-area distribution of first-order pseudopaths (blue dots) for (a) reflection and (b) transmission. Zeroth-order pseudopaths (i.e., the classical paths) are denoted by orange triangles (same as Fig. 2). The number of first-order pseudopaths up to length $L=40$ is larger by more than a factor of 60 compared to the number of classical paths.

equal length. The change in the branch structure is more dramatic in the spectrum of transmitted paths [Fig. 3(b)]. The pseudopaths lead to a "symmetrization" of the length-area distribution. The symmetrization results primarily from paths which change their rotational direction through diffractive scattering [see the pseudopath in the inset of Fig. 3(b)]. Branches of classical paths are now completed by symmetric pseudopath "partner" branches of approximately equal length and different enclosed area.

The weight and the phase of interfering paths are strongly influenced by the diffraction coefficient $v\left(\theta^{\prime}, \theta, k, d\right)$. As will be demonstrated in Sec. V, the previously employed Fraunhofer theory of diffraction ${ }^{12-15}$ is not sufficiently accurate as to give quantitatively reliable results for transport properties. The same holds for the Kirchhoff theory of diffraction ${ }^{10}$ which is closely related to the Fraunhofer theory of diffraction and gives similar results for the diffraction coefficients (see Fig. 5 in the following section). For a quantitative agreement between the PSCA and quantum mechanics, it is thus necessary to go beyond low-order diffraction approximations and use a more sophisticated theory of diffraction. We present such a theory in the following section. 


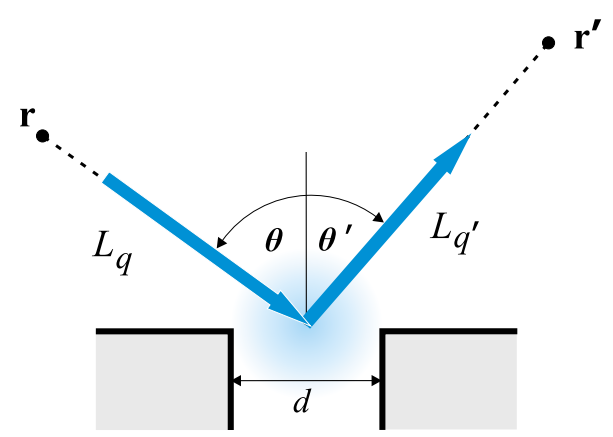

FIG. 4. (Color online) Sketch of diffraction at an open lead mouth: a path $q$ reaches the orifice under an angle $\theta$ and is backscattered into a path $q^{\prime}$ that leaves with an angle $\theta^{\prime}$ (the angles $\theta$ and $\theta^{\prime}$ as depicted in the figure have opposite signs). The dashed lines denote that $\vec{r}$ and $\vec{r}^{\prime}$ are in the far-field region.

\section{DIFFRACTION AT THE LEAD}

The contribution of a given classical path (within the circular billiard) with path length $L_{q}$ to the standard semiclassical Green's function, $G^{\mathrm{SCA}}$, is

$$
G_{q}^{\mathrm{SCA}}=\frac{1}{\sqrt{2 \pi k L_{q}}} e^{i k L_{q}-i 3 \pi / 4-i \pi / 2 \mu_{q}}
$$

This equation is also valid for the propagation in free space. Equation (12) is the basic building block entering the Dyson Eq. (10) together with the diffraction coefficient $v\left(\theta^{\prime}, \theta, k, d\right)$. In line with the far-field approximation underlying diffraction theory, $v\left(\theta^{\prime}, \theta, k, d\right)$ is assumed to be independent of the length of the path approaching or exiting the diffractive scattering region.

A successful application of the pseudopath semiclassical approximation outlined in the preceding subsection requires the determination of accurate diffraction coefficients $v\left(\theta^{\prime}, \theta, k, d\right)$. Different approximations have been used in the past for the inclusion of diffraction effects: Kirchhof diffraction approximation (KDA), ${ }^{10}$ Fraunhofer diffraction approximation (FDA), ${ }^{12-15}$ GTD, ${ }^{32}$ and the UTD. ${ }^{30,31}$ We have developed a theory for the reflection at open lead mouths by combining the GTD with the UTD (the GTD-UTD) to take into account paths that scatter multiple times between the edges of the leads (see Section 1 of the Appendix). With this theory we obtain diffraction coefficients in excellent agreement to quantum mechanics.

Consider, as a test case, the diffractive scattering (Fig. 4) at the lead mouth described by the first-order term [Eq. (11)],

$$
G_{q^{\prime}, q}^{\mathrm{PSCA}^{(1)}}=G_{q^{\prime}}^{\mathrm{SCA}} v\left(\theta^{\prime}, \theta, k, d\right) G_{q}^{\mathrm{SCA}} .
$$

The incoming path $q$ is incident at angle $\theta$ (measured with respect to the surface normal) and is diffractively scattered into angle $\theta^{\prime}$ under which path $q^{\prime}$ leaves the scattering region. We compare (Fig. 5) the present GTD-UTD theory with the FDA, the KDA, and exact quantum-mechanical (QM) calculations. ${ }^{10}$ Even for a typical $k$ value in the lowmode regime $(k=2.5 \pi / d)$, the agreement between the GTDUTD and the exact QM calculations is very good whereas both the FDA and the KDA display clear deviations from the

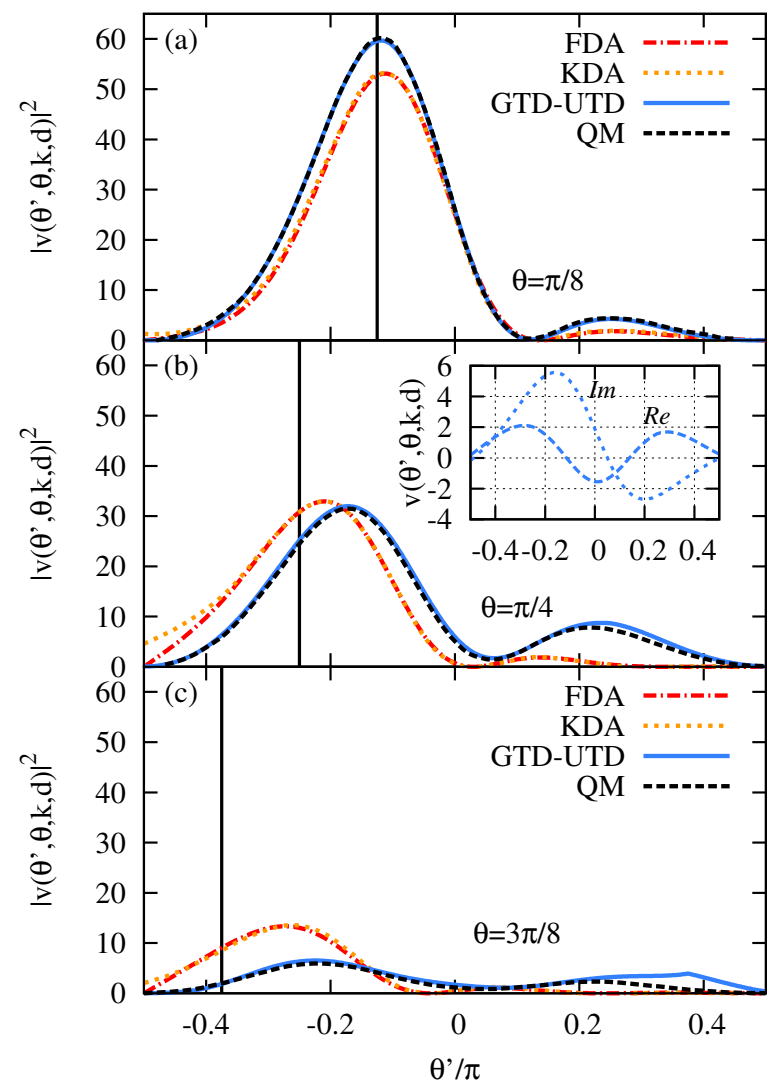

FIG. 5. (Color online) Absolute square of the diffraction coefficient $\left|v\left(\theta^{\prime}, \theta, k, d\right)\right|^{2}$ for diffractive scattering (see Fig. 4) within the FDA, the KDA, the GTD-UTD, and exact quantum-mechanical data for angle of incidence (a) $\theta=\pi / 8$, (b) $\theta=\pi / 4$, and (c) $\theta$ $=3 \pi / 8$ at $k=2.5 \pi / d$. The vertical lines in each frame mark the angles of specular reflection. The quantum-mechanical (QM) and KDA coefficients are taken from Ref. 10. Inset of Fig. (b): the real and imaginary parts of $v\left(\theta^{\prime}, \theta, k, d\right)$ within the GTD-UTD.

QM values. While these deviations do not appear dramatic at first glance, they are, in fact, quantitatively very important as the diffraction coefficient enters the Dyson series [Eq. (10)] to all orders. Note, however, that the GTD-UTD would fail in the limit $\theta \rightarrow \pi / 2$ [as indicated by the kink at $\theta^{\prime}=\theta$ $=3 \pi / 8$ in Fig. 5(c)]. This deficiency is of no concern for the present applications as the probability for diffractive scattering tends to zero in this limit. The maximum of the diffractive reflection probability is clearly around the specular value $\theta^{\prime} \approx-\theta$. However, it is important to note that the probability distribution possesses a local maximum at the backscattering angle $\theta^{\prime} \approx \theta$. A non-negligible part of the electron wave is backscattered into the direction from where it came from. This diffractive back reflection should not be confused with the well-known Andreev back reflection in which the backreflected particle simultaneously undergoes a particle-hole exchange..$^{42}$ Back-reflected paths are responsible for the symmetrization of the distribution of transmitted paths [Fig. 3(b)] and are crucial for the understanding of the weak-localization dip in the transmission. ${ }^{16}$

While the KDA and the FDA have been successfully used in the past to explain certain features of conductance fluctuations, ${ }^{10,12-15}$ only the GTD-UTD is precise enough to 


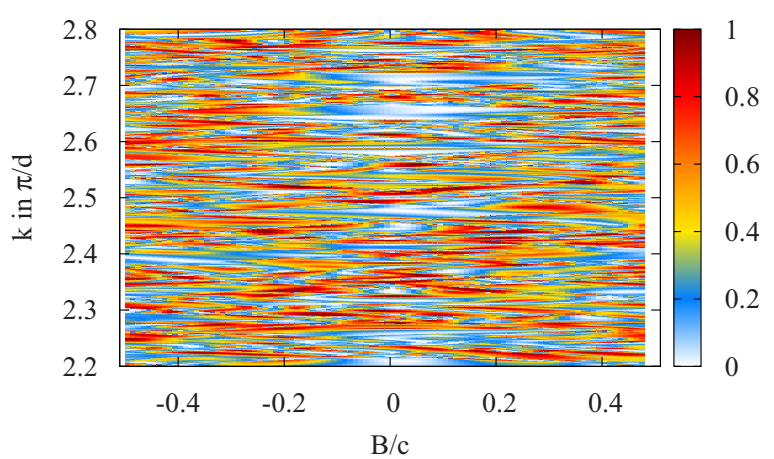

FIG. 6. (Color online) Two-dimensional distribution of the absolute square of the quantum $S$-matrix element $\left|t_{22}(k, B)\right|^{2}$ $=T_{22}(k, B)$ as a function of the wave number $k$ and the magnetic field $B$.

reproduce transport semiclassically on a quantitative level (see Sec. V). In Sec. V B we compare the results for transport properties obtained by implementing the GTD-UTD and the FDA to full quantum-mechanical calculations.

\section{PATHS IN QUANTUM TRANSPORT}

The information on paths governing quantum transport can be reliably extracted from the two-dimensional Fourier
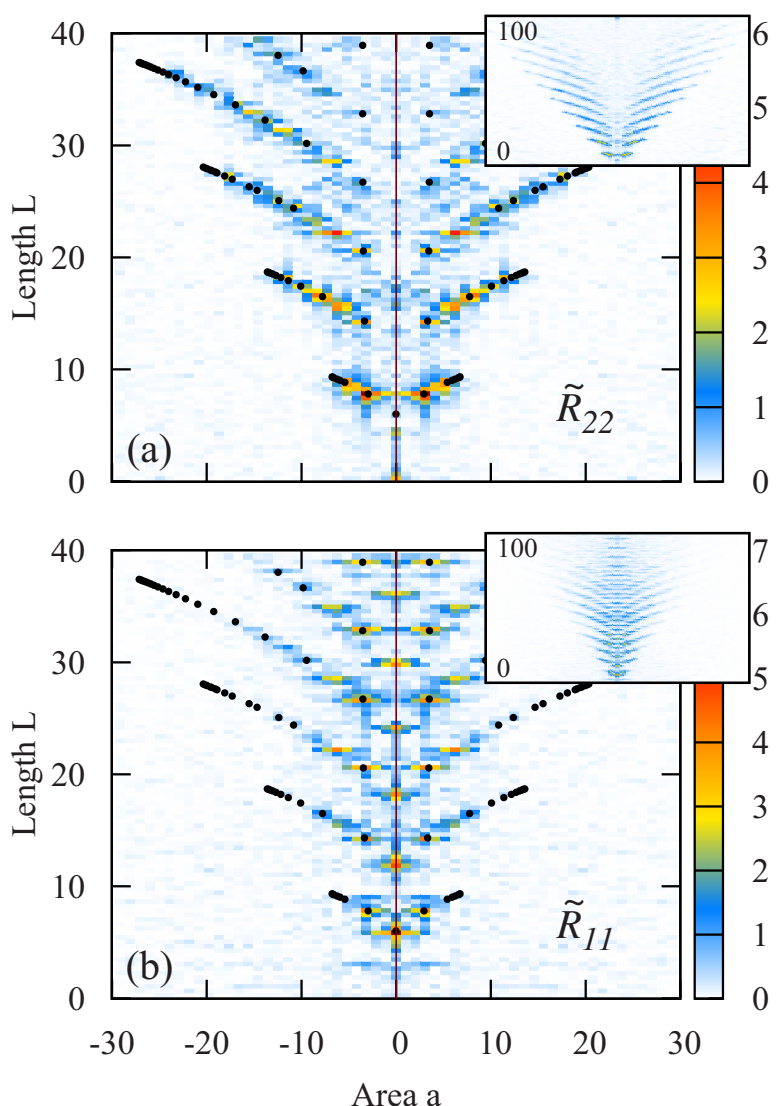

transforms of the quantum-mechanical $S$-matrix elements, ${ }^{16}$ $S_{n m}(k, B)$. The $S$-matrix elements display a strongly fluctuating pattern as a function of $k$ and $B$ [see Fig. 6 for $T_{22}(k, B)$ ]. Since the canonically conjugate variables to the wave number $k$ and the magnetic field $B$ are the length $L$ and the directed area $a$, respectively, the Fourier transform

$$
\tilde{S}_{n m}^{j, i}(L, a)=\int d k \int d B e^{-i(k L+B / c a)} S_{n m}^{j, i}(k, B)
$$

allows the unambiguous identification of quantum paths contributing to quantum transport via their length and enclosed area [Figs. $7(\mathrm{a})-7(\mathrm{~d})]$. No a priori assumption as to the existence of classical paths $q(L, a)$ with path length $L$ and area $a$ enters Eq. (14). The two-dimensional path-length-area spectrum allows to identify both classical as well as nonclassical contributions to the full quantum spectra. Figure 7 displays examples of path-length-area spectra $\left|\tilde{S}^{i j}(L, a)\right|^{2}$. [Note that Fig. 7(c) is the absolute square of the Fourier transform of the transmission amplitude whose absolute square is plotted in Fig. 6]. Obviously the strong fluctuations of conductance in quantum transport (Fig. 6) are the result of the interference of clearly identifiable (quantum) paths (Fig. 7).

The quantum-mechanical $S$-matrix elements $S_{n m}(k, B)$ are determined with the help of the modular recursive Green's-
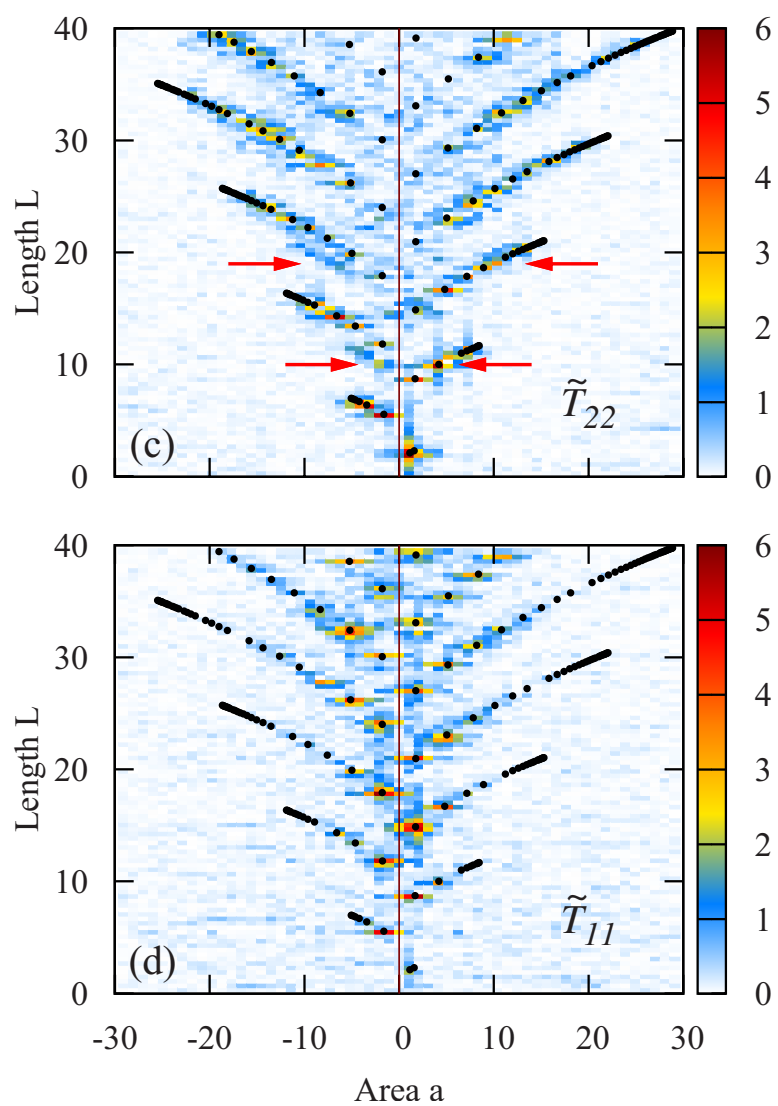

FIG. 7. (Color online) The absolute square of the Fourier transform $|\tilde{S}(L, a)|^{2}$ as a function of length $L$ and enclosed directed area $a$ : (a) $\widetilde{R}_{22}(L, a)$, (b) $\widetilde{R}_{11}(L, a)$, (c) $\widetilde{T}_{22}(L, a)$, and (d) $\widetilde{T}_{11}(L, a)$. The color shading is determined by $\log \left(|\widetilde{S}(L, a)|^{2}+1\right)$. The insets show the probabilities along the entire resolved length. The integration of Eq. (14) is performed numerically over $k \in[2.2,3.45] \pi / d$ discretized with 251 points and $B / c \in[-3,3]$ with 121 points. The spectra are compared to the distribution of classical paths (black dots) at zero $B$ field (the curvature of the trajectories in the present magnetic field and energy regime is negligible). 
(a)

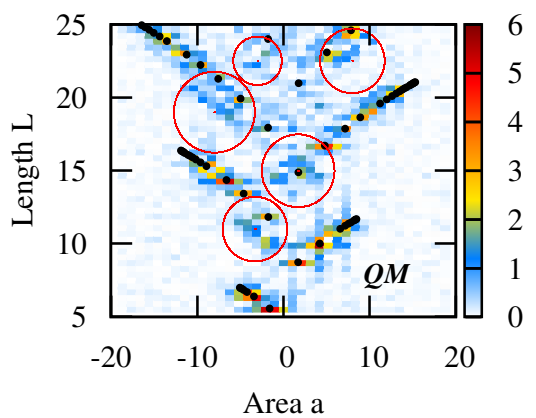

(b)

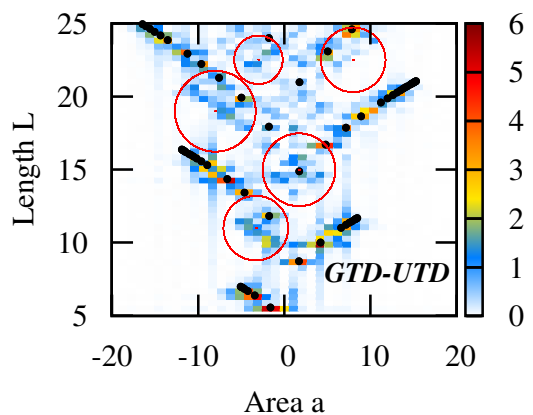

(c)

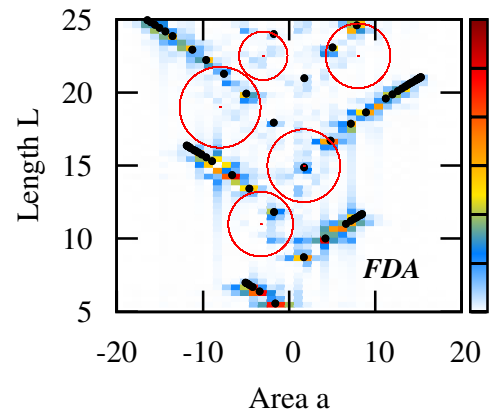

FIG. 8. (Color online) $\widetilde{T}_{22}(L, a)$ as a function of length $L$ and enclosed directed area $a$ : (a) full quantum $S$-matrix, (b) the PSCA using the GTD-UTD, and (c) the PSCA using the FDA for the diffraction coefficients. Several regions with nonclassical paths are highlighted by red circles.

function method m,35 $^{34,}$ and then numerically Fourier transformed. Finite discretized intervals must be used when performing the Fourier transform numerically. The integration intervals are denoted by $\Delta k=k_{\max }-k_{\min }\left(\Delta B=B_{\max }-B_{\min }\right)$ and the numerical grid spacings by $\delta k(\delta B)$. Accordingly, the resolvable length is $\Delta L=2 \pi / \delta k$ and the resolvable area interval is $\Delta a=2 \pi /(\delta B / c)$. The magnitude of the $S$-matrix elements decreases with increasing length, which is an obvious consequence of open systems: the probability to stay within the cavity decreases with increasing length. The parameter $\delta k$ must be chosen sufficiently small such that the maximum resolvable length $(\Delta L)$ lies already in the region of strongly reduced amplitudes. Otherwise, the Fourier spectrum is visibly backfolded onto the fundamental interval. The magnetic field interval is further restricted by the requirement that the curvature of the paths is negligible, i.e., the cyclotron radius $c k / B$ should be much larger than the circle radius $\rho$. We chose the interval $\Delta k$ and $\Delta B$ as well as the number of interval points such that a maximum length of $\Delta L=100$ is resolved [in Figs. 7(a)-7(d) only the contributions with $L$ $\leq 40$ are shown, the insets contain the entire spectrum]. Except for $R_{11}$, the absolute square of the $S$-matrix elements is considerably damped at a length of $\Delta L=100$. Thus, the graphs represent essentially the entire length-area spectrum. Only for $R_{11}$, contributions with $L>100$ are non-negligible which leads to a backfolding near $L=0$ [the lowest branch structure near $L=2$ in Fig. 7(b)].

The quantum length-area spectra provide detailed information on the paths contributing to transport through a specific system. They represent the paths entering the full Feynman path integral. The following general trends can be observed. Long paths are more prevalent in $S$-matrix elements connecting low mode numbers (in the present case they contribute more strongly to $S$-matrix elements $\widetilde{S}_{11}$ than to $\widetilde{S}_{22}$ ). Lower modes favor smaller entrance and exit angles that are associated with longer paths with a larger number of bounces off the cavity boundary. The most important observation is the remarkably close correspondence of the quantum-mechanical length-area spectrum to its classical counterpart. Important contributions are located near classical paths. Moreover the branch structure of classical paths is reproduced [Figs. $7(\mathrm{a})-7(\mathrm{~d})]$. On the other hand, there are distinct structures which do not correspond to classical paths and which can be identified using the distributions in Figs. 3(a) and 3(b). The quantum-mechanical length-area spectra confirm the existence and substantial role of nonclassical paths, the pseudopaths: these are those paths that are one or several times diffractively reflected off the lead before exiting the cavity. Two examples are given in the following: for $\widetilde{T}_{22}$ we identify an ensemble of diffractive paths which, among others, contribute to a symmetrization of the spectrum [nonclassical branches in Fig. 7(c), two of them together with the classical partner branches are marked by arrows]. $R_{11}$ reveals the importance of paths that are geometrically reflected off the open lead (e.g., the periodic contributions near $a=0$ along the length axis belong to horizontal paths bouncing increasingly many times back and forth).

The importance of a given class of paths to quantum transport can be delineated by inverting this decomposition process. Deleting a selected class of paths (classical or nonclassical) from $\widetilde{S}_{i j}(L, a)$ and performing the inverse Fourier transform gives rise to truncated $S$-matrix elements $\bar{S}_{i j}(k, B)$ from which certain path contributions have been removed in a controlled manner. This is the key to detailed quantitative tests of semiclassical theories. Since summation of the (P)SCA over arbitrarily long paths is prohibitively complicated we can compare truncated quantum and semiclassical $S$-matrix elements where both quantum and classical paths only up to a maximum path length $L \leq L_{\max }$ are included. The length-area spectra also allow sensitive tests for the proper diffractive weight of a given class of paths in a semiclassical theory. To this end, we first calculate the $S$-matrix elements within the PSCA and then perform the Fourier transform [Eq. (14)] in analogy to quantum calculations. To analyze the role of a proper diffraction coefficient we use either the GTD-UTD (which gives good agreement with quantum mechanics, see Fig. 5) or the FDA (with poor agreement with quantum mechanics, see likewise Fig. 5). The fact that back reflection into the cavity is poorly described within the FDA is mirrored in the semiclassical length-area spectrum where important nonclassical (diffractive) contributions have a far too low weight [Fig. 8(c)]. In particular, the diffractive change in the rotational direction is insufficiently described (see Fig. 5 for $\theta^{\prime}>0$ ). A clear indication for the essential role of the corresponding paths is the 
improvement within the GTD-UTD. The length-area spectrum within the GTD-UTD remarkably reproduces even fine details of the quantum-mechanical spectrum [compare Figs. 8(a) and 8(b)]. The nonclassical path sets in Fig. 8 can be identified using the path distributions within the PSCA (Fig. 3 ). The length-area spectra do not leave any ambiguity as to which paths contribute by which phase and weight. By taking into account pseudopaths and weighting them with the appropriate diffraction coefficients the quantum-mechanical path spectrum is reproduced on a quantitative level. In the following section we demonstrate how the accurate representation of the length-area spectra within PSCA directly translates into quantitative reproduction of transport properties.

\section{APPLICATION TO TRANSPORT THROUGH REGULAR BILLIARDS}

Phase-coherent ballistic transport governed by quantum interference influences the conductance in several important ways: the conductance strongly fluctuates as a function of the Fermi energy, the magnetic field, or the cavity geometry (conductance fluctuations, CF). The conductance is, on average, suppressed compared to the classical prediction and increases with external magnetic field (weak localization, WL). The noise carries the signatures of quantum-mechanical uncertainty (shot noise).

Path interference has been the key to the understanding of phase-coherent ballistic transport. ${ }^{7-26,43}$ For chaotic systems and large mode numbers quantum-transport properties have been attributed ${ }^{17-23}$ to the interference of classically allowed paths (SCA). We demonstrate in the following for the circular billiard at low mode numbers (accessible to experiments) that $\mathrm{CF}, \mathrm{WL}$, and shot noise cannot be explained by the interference of classical paths alone. In this regime, the SCA overestimates the $\mathrm{CF}$, does not reproduce the weaklocalization dip and also shows poor quantitative agreement with the quantum-mechanical prediction for shot noise. These difficulties can be overcome with the PSCA when a high-level diffraction approximation, the GTD-UTD, is employed. For technical reasons, we perform in both the SCA and the PSCA the summation over paths only up to a maximum cutoff length $L_{\max }$. Correspondingly, we truncate the full quantum scattering matrix elements by setting all elements $\widetilde{S}_{n m}(L, a)$ with lengths exceeding $L_{\max }$ to 0 and carry out the inverse Fourier transform. This allows a quantitative comparison between semiclassical and quantum calculations unaffected by the (inevitable) unitarity deficiency of a truncated semiclassical path sum.

\section{A. Conductance fluctuations}

$\mathrm{CF}$ have been identified as a direct manifestation of phase-coherent transport (see, e.g., Refs. 1, 2, 8, 18, and 44-47, and references therein). The strong fluctuations of the conductance as a function of, e.g., energy originate from path interference and thus give evidence for the wave nature of electrons in quantum dots. The CF offer one of the most stringent testing grounds for a semiclassical theory, when good agreement on the level of each individual $S$-matrix element is required.

In the following, we compare the results for $\mathrm{CF}$ within the PSCA with the GTD-UTD, the SCA, and the quantummechanical calculations as a function of $k$ at vanishing magnetic fields $B=0$. Results for $B \neq 0$ averaged over $k$ can be found in Sec. V B.

The semiclassical and quantum-mechanical results both display strong fluctuations of the conductance [i.e., the total transmission $T(k, B=0)$ ]. Their amplitude is, however, extremely sensitive to any deficiencies in the semiclassical approximations (inappropriate weighting of paths, missing paths). We emphasize that the comparison of Fig. 9 is on a fully differential level. No energy or ensemble average is involved. Unsurprisingly, the agreement between the SCA and the quantum data is poor and on a level of qualitative agreement at best [Figs. 9(a) and 9(b)]. The functional dependence of $T(k, B=0)$ and $R(k, B=0)$ seems only weakly related to the quantum-mechanical prediction. By ensemble averaging (e.g., over a suitable $k$ interval) these discrepancies would be (partially) removed (or masked). To correctly reproduce the fluctuations in $T(k, B=0)=\sum_{m, n}^{M}\left|t_{m n}(k, B=0)\right|^{2}$, all individual mode-to-mode amplitudes $t_{m n}$ have to be accurate. Obviously, the contribution of pseudopaths included in the PSCA but missing in the SCA significantly improves the agreement with the quantum conductance fluctuations [Figs. 9(c) and 9(d)].

Even for averaged quantities, such as the averaged conductance $\langle T\rangle_{\Delta k}$ and the variance of the conductance fluctuations $\sigma=\sqrt{\left\langle T^{2}\right\rangle_{\Delta k}-\langle T\rangle_{\Delta k}^{2}}$, the deficiencies of the SCA are still visible, in particular, at larger cutoff lengths $L_{\text {max }}$. Both $\langle T\rangle_{\Delta k}$ and $\sigma$ are overestimated (Fig. 10) as compared to quantum mechanics which can be attributed, in part, to the lack of correlation among purely classical paths.

In contrast, the PSCA shows very good agreement with quantum mechanics for both the averaged conductance as well as for the variance. For averaged quantities (Fig. 10) the inclusion of up to fourth-order diffractive scattering for $L_{\text {max }}=40$ is sufficient while for fully differential quantities (Fig. 9) fifth-order corrections for $L_{\max }=40$ still improve the agreement.

We point out that for the present system, taking into account paths up to a length of $L_{\max }=50$ which corresponds to 16 radial traversals through the billiard, one reaches $70 \%$ of the unitarity level within both the truncated quantum mechanics and the PSCA. The essential contributions to $\mathrm{CF}$, WL, and shot noise (see the following sections) is thus rooted in this subset of relatively short paths.

\section{B. Weak localization}

WL is a well-known quantum correction to the classical diagonal approximation (see, e.g., Refs. 1, 2, 8, 16-20, 28, and 48-52, and references therein) where the later corresponds to the restriction to terms $q=q^{\prime}$ in the double sum over paths when calculating $\left|t_{n m}^{\mathrm{SCA}}\right|^{2}$ from Eq. (7). Offdiagonal terms $q \neq q^{\prime}$ give rise to quantum interferences which manifest themselves as an increase in the averaged total reflection $\langle R(B)\rangle_{\Delta k}=\Sigma_{m, n}\left\langle R_{n m}(B)\right\rangle_{\Delta k}$ at $B=0$ in form of a 
(a) Reflection

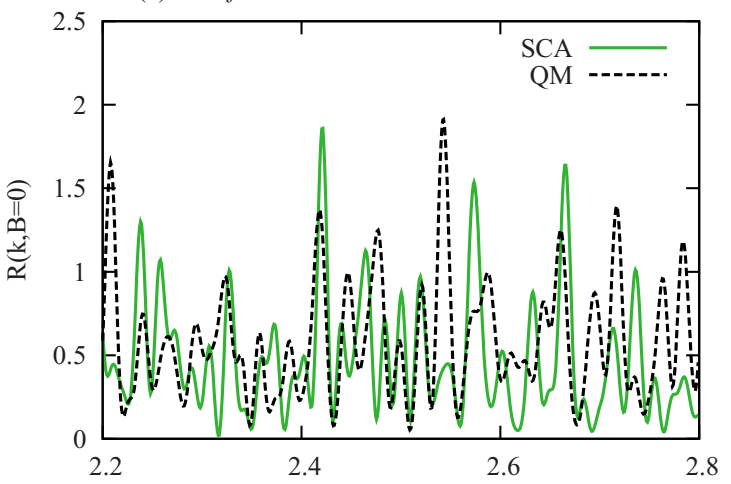

(b) Transmission

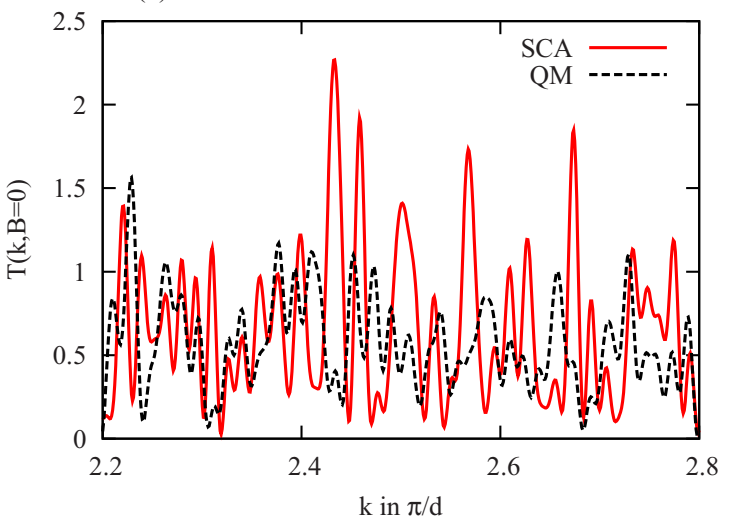

(c) Reflection

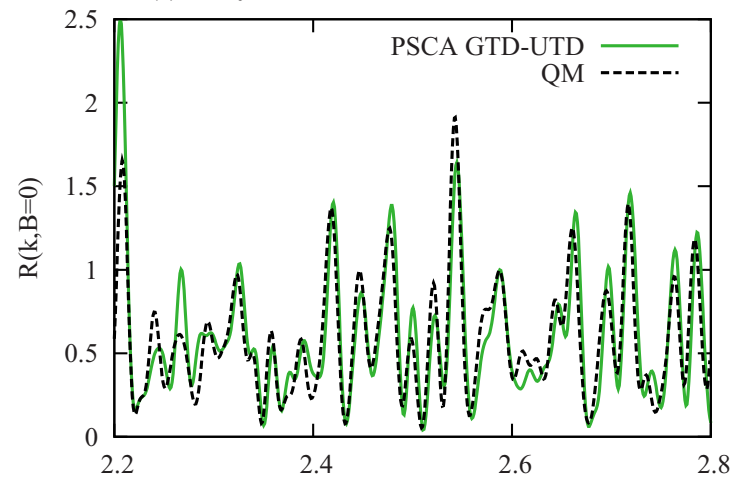

(d) Transmission

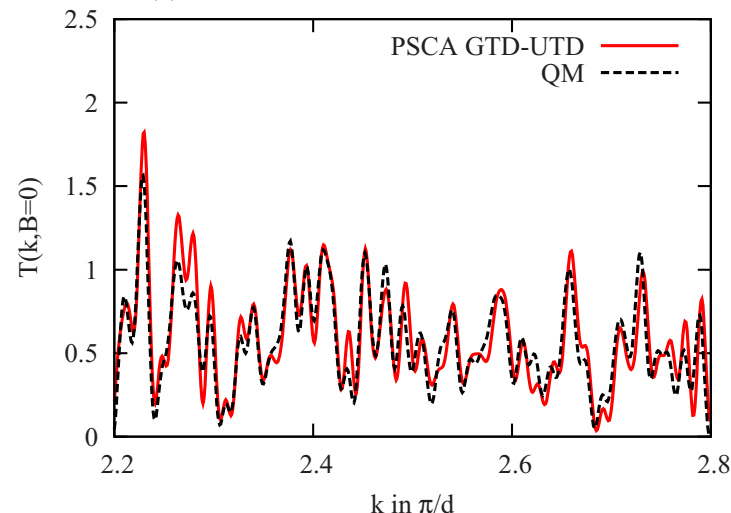

FIG. 9. (Color online) (Conductance) Fluctuations in the transmission $T(k, B=0)$ and reflection $R(k, B=0)$ as a function of $k$. The maximal included length is $L_{\max }=40$. (a) Reflection and (b) transmission within the SCA (red/green solid line), and quantum mechanics (black dashed line). For the diffraction coefficients $c_{m}(\theta, k, d)$ entering the SCA we have used the GTD-UTD. (c) Reflection and (d) transmission within the PSCA with the GTD-UTD (red/green solid line), and quantum mechanics (black dashed line). The PSCA includes diffractive scattering up to fifth order.

pronounced peak. Correspondingly, the averaged total transmission $\langle T(B)\rangle_{\Delta k}=\sum_{m, n}\left\langle T_{n m}(B)\right\rangle_{\Delta k}$ features a dip which is an immediate consequence of unitarity. For the investigation of the weak-localization dip (peak) we employ an average over a small window $\Delta k=[2.2-2.8] \pi / d$ of the $k$ dependence of the probabilities: $\left\langle T_{n m}(B)\right\rangle_{\Delta k}=\int_{\Delta k} d k\left|t_{n m}(k, B)\right|^{2} \quad$ and $\left\langle R_{n m}(B)\right\rangle_{\Delta k}=\int_{\Delta k} d k\left|r_{n m}(k, B)\right|^{2}$.

Since semiclassical theories are, by construction, not necessarily unitary at a given level of approximation, the anti-

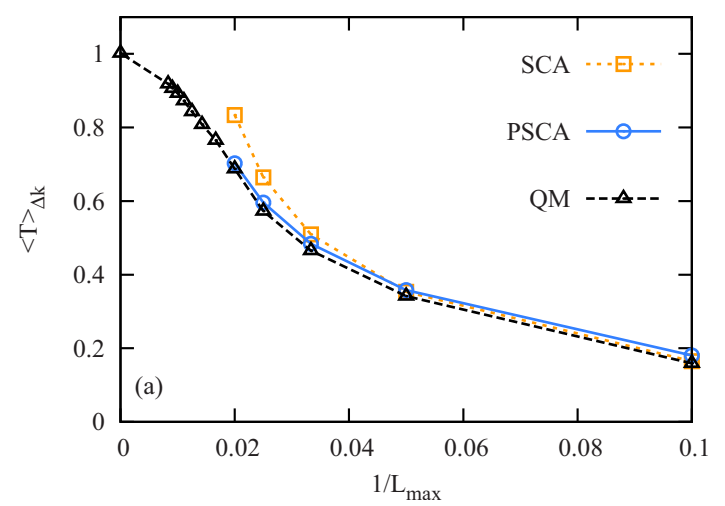

correlated peak-dip structure near $B=0$ provides a sensitive test for semiclassical approximations. It has been shown that the quantum anticorrelation between reflection and transmission $\left[\langle R(B)\rangle_{\Delta k}-\langle R(B=0)\rangle_{\Delta k}\right]=-\left[\langle T(B)\rangle_{\Delta k}-\langle T(B=0)\rangle_{\Delta k}\right]$ requires a correlation of transmitted and reflected paths. ${ }^{16}$ This correlation is absent in the SCA such that no transmission dip is reproduced [see Fig. 11(b)].

The role of diffraction in a quantum billiard manifests itself by a very good agreement of the PSCA with quantum-

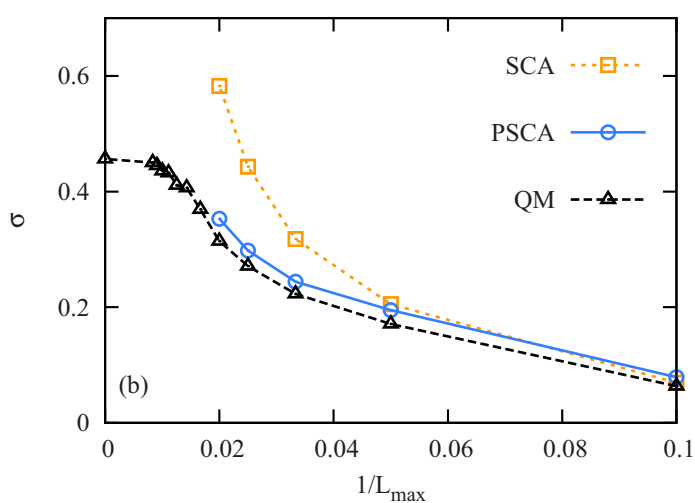

FIG. 10. (Color online) Comparison between the PSCA, the SCA, and quantum mechanics (QM) for (a) the averaged transmission $\langle T\rangle_{\Delta k}$ as a function of the inverse cutoff length $1 / L_{\max }$ and (b) the averaged variance of the conductance fluctuations $\sigma$. The average is performed in the interval $k \in[2.2,2.8] \pi / d$. The data within the PSCA are calculated in third order for lengths $L_{\max }=10-30$, in fourth order for $L_{\text {max }}$ $=40$ and fifth order for $L_{\max }=50$. The QM results extend to the exact value $\left(L_{\max }=\infty\right)$. 

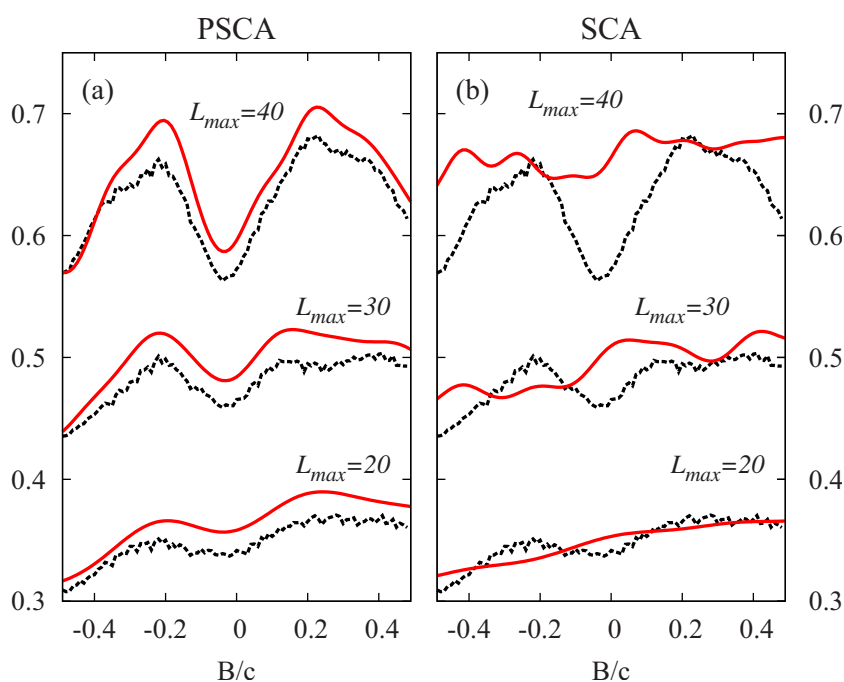

FIG. 11. (Color online) Comparison between truncated quantum mechanics, the SCA, and the PSCA for the weak-localization dip in transmission for different $L_{\max }$. (a) PSCA using the GTD-UTD for all diffraction coefficients (red solid line). (b) Standard SCA (red solid line) (using the GTD-UTD for the diffraction coefficients $c_{m}(\theta, k, d)$ for entering and exiting the circular cavity.) Black dashed lines: quantum results.

mechanical results [see Fig. 11(a)]. Tests for WL as a function of the cutoff length $L_{\max }$ of the paths as well as of the order of diffractive scattering included show that convergence toward the (truncated) quantum result is reached for $L_{\max }=40$ when diffraction up to fourth order is included. We note an improvement compared to previous third-order calculations ${ }^{16}$ especially due to the inclusion of the (small) real part of the diffraction coefficient $v\left(\theta^{\prime}, \theta, k, d\right)$.

It is instructive to analyze the build up of the weaklocalization peak in reflection and of the dip in transmission from individual $S$-matrix elements. In reflection only the diagonal elements $R_{11}(B)$ and $R_{22}(B)$ show a peak while the off-diagonal elements $R_{12}(B)$ and $R_{21}(B)$ exhibit a dip [Figs. $12(\mathrm{c})$ and $12(\mathrm{~d})]$. This is due to the fact that time-reversal symmetric paths contributing to $R_{n m}(B)$ with different parity of modes $m$ and $n$ acquire an additional phase shift of $\pi$. As an example consider $R_{21}(B)$, entering in mode $m=1$ and exiting in mode $m=2$ produces a phase shift of $\pi$. Thus the time-reversal symmetric paths interfere destructively.

In transmission the major contribution to a dip originates from $T_{22}(B)$ in the chosen energy window of two open modes [Figs. 12(e) and 12(f)]. Note that $T_{21}(B)=T_{12}(B)$ because of the Onsager relation $t_{n m}^{(2,1)}(k, B)=t_{m n}^{(1,2)}(k,-B)$ and the symmetry of the circular billiard which ensures that $t_{m n}^{(1,2)}(k$, $-B)=t_{m n}^{(2,1)}(k, B)$. Due to time-reversal symmetry, $R(B)=R($ $-B)$ giving rise to a symmetric peak in $R$ as a function of $B$ (Fig. 12). For unitary transport, $T(B)=1-R(B)$, which implies a symmetric dip in transmission. For the truncated quantum mechanics and semiclassics where long paths are omitted unitarity is not preserved and $T(B)$ is not exactly symmetric but features a slight shift of the minimum toward $B<0$.

Overall, the agreement between the PSCA and the full quantum calculation (truncated at the same path length) is

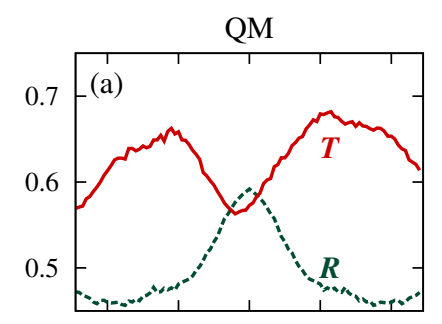

PSCA with GTD-UTD
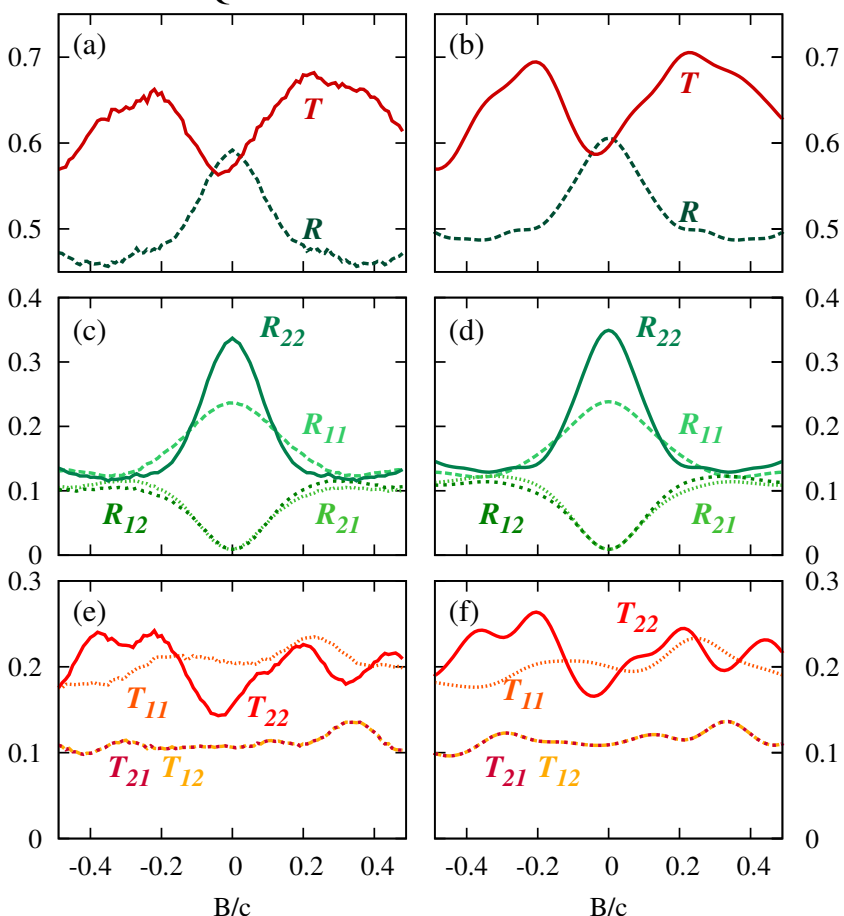

FIG. 12. (Color online) Weak localization for $L_{\max }=40$ as produced by quantum mechanics: left column (a), (c), and (e), and PSCA with the GTD-UTD: right column (b), (d), and (f).

remarkable (Fig. 12). The residual minor deviations are mainly ascribed to deficiencies in the diffraction coefficients for which we use an analytical far-field approximation (Sec. III and Appendix). As, e.g., the diffraction coefficients $c_{m}(\theta, k, d)$ enter $R_{n m}, T_{n m}$ to the fourth power, a small deficiency in $c_{m}(\theta, k, d)$ can have a sizeable effect on $R_{n m}$ and $T_{n m}$. The offset of the total reflection $R$ and transmission $T$ within PSCA [Fig. 12(b)] compared to the quantummechanical result [Fig. 12(a)] mainly originates from imperfections in $R_{22}$ and $T_{22}$ [compare Figs. 12(c)-12(f)]. The imperfection could be cured by including a correction factor of $\approx 0.97-0.99$ in $c_{2}(\theta, k, d)$.

To demonstrate how sensitively transport properties depend on the weights of the contributing paths we show the results for weak localization calculated within PSCA but now using the FDA instead of the GTD-UTD for the diffraction coefficients (Fig. 13). (We have used third order of the PSCA here since the deviation between third and fourth order is much smaller than the errors due to the simpler diffraction theory.) It is striking that especially both $R_{22}$ and $T_{22}$ seem to be underestimated which is due to deficiencies in $c_{2}(\theta, k, d)$ within the FDA. It is worthwhile pointing out that the FDA diffraction could not be "repaired" by a correction factor, as the errors in $R_{22}$ and $T_{22}$ are different. In other words, transmission is more "sensitive" to a correct implementation of pseudopaths than reflection. Of particular importance are paths which change the rotational direction. ${ }^{16}$ Since the FDA does not give sufficiently weight to this class of pseudopaths (see Fig. 5) the transmission dip cannot be well reproduced. 

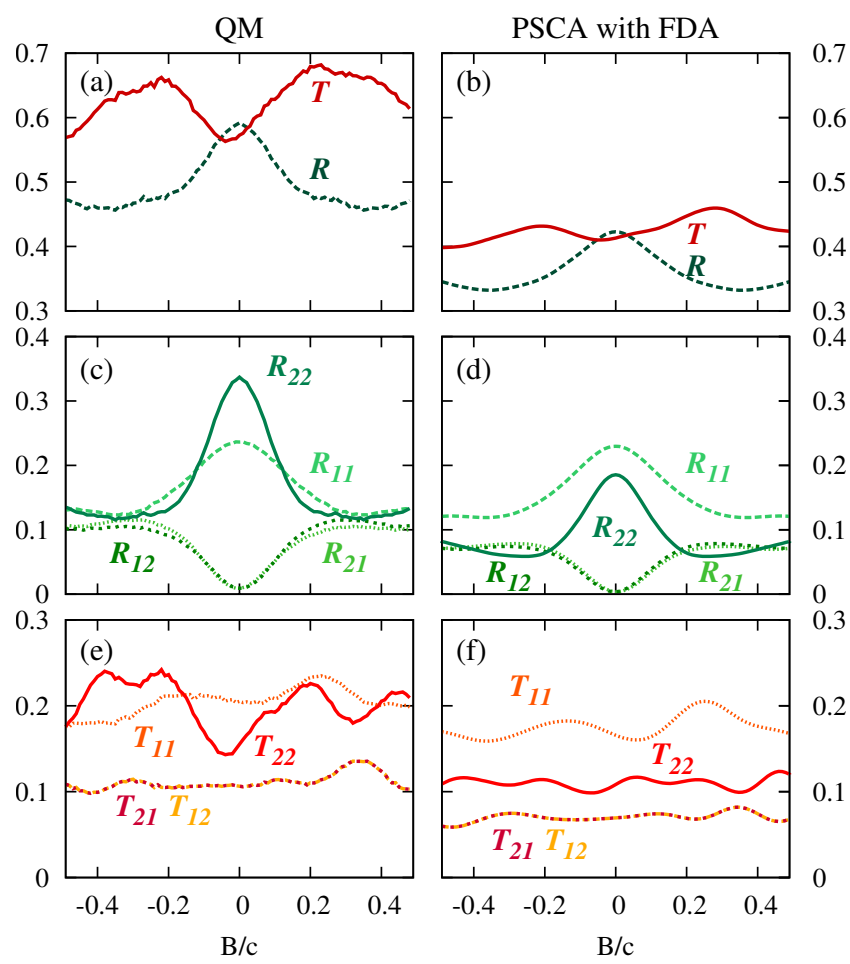

FIG. 13. (Color online) Weak localization for $L_{\max }=40$. Left column (a), (c), and (e): quantum calculations, right column (b), (d), and (f): PSCA with diffraction coefficients from FDA.

\section{Shot noise}

Another quantity characteristic for quantum transport is the quantum shot-noise power of the current (see, e.g., Refs. 1, 18, 21, 45, and 53-60, and references therein). At zero temperature $(T=0)$, the time-dependent current noise is due to the granularity of the electron charge and carries information about the wave vs particle nature of charge transport. The Fano factor $F$ measures the amount by which the noise in phase-coherent transport is suppressed relative to the Poissonian value of uncorrelated classical electrons. Within the Landauer-Büttiker picture, $F$ can be expressed as ${ }^{53}$

$$
F=\frac{\left\langle\operatorname{Tr}\left(t^{\dagger} t r^{\dagger} r\right)\right\rangle_{\Delta k}}{\left\langle\operatorname{Tr}\left(t^{\dagger} t\right)\right\rangle_{\Delta k}}=\frac{\left\langle\sum_{n} \tau_{n} \eta_{n}\right\rangle_{\Delta k}}{\left\langle\sum_{n} \tau_{n}\right\rangle_{\Delta k}}
$$

with $\tau_{n}$ and $\eta_{n}$ being the eigenvalues of the Hermitian matrices $t^{\dagger} t$ and $r^{\dagger} r$, respectively.

Calculating the shot-noise power from nonunitary scattering matrices is obviously a delicate matter, as replacement of $r^{t} r$ by $1-t^{t} t$ leads, unlike for unitary descriptions, to different results. Furthermore, such a replacement may result in negative and thus unphysical values for the shot-noise power, as nonunitary scattering matrices allow for the possibility of having $\tau_{n}>1$ such that $\left(1-\tau_{n}\right)<0$ (for very high mode numbers as, e.g., in Ref. 18 such a situation may, however, be unlikely). By using Eq. (15), such difficulties can be avoided as both the transmission and reflection eigenvalues $\tau_{n}, \eta_{n}$ are, by construction, real and positive not only for the truncated

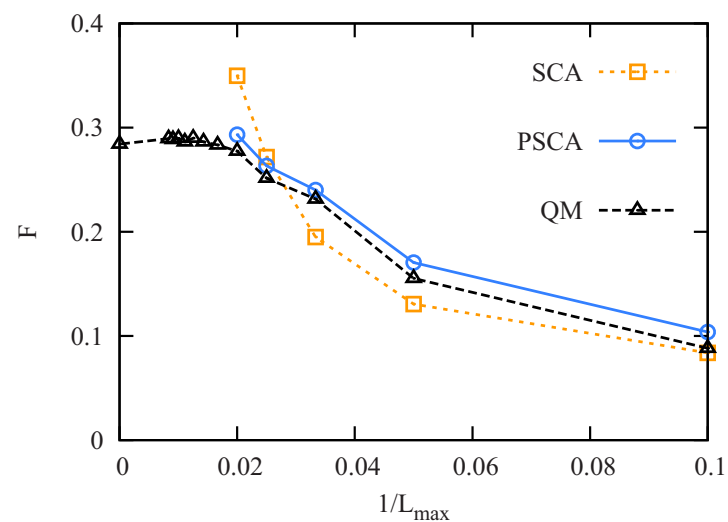

FIG. 14. (Color online) The $k$-averaged Fano factor $F$ as a function of the inverse cutoff length $L_{\max }$. The average is performed over the interval $k \in[2.2,2.8] \pi / d$. Comparison between PSCA with the GTD-UTD, the SCA, and QM. ( $L_{\max }=\infty$ corresponds to the exact result.)

quantum calculation but also for the PSCA and the standard SCA. The standard SCA result for $F$ strongly deviates from the quantum-mechanical data (see Fig. 14). For small cutoff lengths $L_{\max }$, the value of $F$ is smaller but increases more rapidly than the quantum-mechanical result with $L_{\max }$. The PSCA yields very good agreement with the quantummechanical result for the shot-noise Fano factor $F$. Note that for the largest cutoff length $L_{\max }=50$ the Fano factor $F$ is already converged to its asymptotic value $F \approx 0.28$ suggesting that long paths do not play a significant role for $F$. This result agrees with the finding ${ }^{54,55}$ that the shot-noise power is of similar magnitude for regular and chaotic billiards as differences in the dynamics are most strongly felt by very long paths.

\section{SUMMARY}

We have presented a semiclassical theory which is able to quantitatively reproduce full quantum results for scattering through microstructures with a specific geometry, in the present case a circular-shaped billiard with leads oriented $90^{\circ}$ relative to each other. The present approach does not invoke the limit of large mode numbers, (where the de Broglie wavelength $\lambda$ is small relative to the lead width) but requires $\lambda$ to be small only on the scale of the linear dimension of the microstructure (the circle). This nonasymptotic semiclassical theory allows a direct comparison with quantum calculations as well with experiments on a systemspecific level for individual $S$-matrix elements avoiding any ensemble averaging or fit parameters. This level of agreement allows us to perform detailed semiclassical investigations of quantum-transport quantities such as the conductance fluctuations, the weak localization, and the shot noise. Our studies show unambiguously that for reproducing these quantities correctly, two major ingredients are, indeed, crucial: (1) the inclusion of "pseudopaths" in the semiclassical propagator which are diffractively backscattered from the interior side of the cavity openings and (2) a sufficiently accurate description of the diffraction coefficients for the injec- 
tion, ejection, and back reflection of particle flux at the cavity openings. We meet the latter requirement by developing a combined geometric and uniform theory of diffraction (GTD-UTD). Pseudopaths are crucial for reproducing the conductance fluctuations in transport and lead to a reduction in its variance. Also for the weak-localization effect, we find that pseudopaths are crucial, as no signature of weak localization appears in the transmission through the circular cavity without their contribution (even when the advanced diffraction theory is employed for all truly classical paths). For the shot-noise power we showed that a standard semiclassical calculation (without pseudopaths) gives sizeable discrepancies. The inclusion of pseudopaths leads to agreement with the quantum-mechanical result. We emphasize that the parameter regime in which we have identified the above effects of pseudopaths coincides with the typical situation in quantum-transport experiments. The latter usually feature only a few open lead modes $M$.

The present results raise several interesting questions for future investigations: the comparison between the semiclassical approximations (PSCA and SCA) and full quantum calculations were performed for truncated path sums up to a finite path length $L \leq L_{\max }$. The primary reason for the truncation was technical, as the number of diffractive pseudopaths exponentially proliferates with $L \rightarrow \infty$ also for classically regular structures and exact path sums become prohibitively difficult to perform. There is, however, a second conceptual motivation. In the experiment, decoherence due to inelastic scattering limits phase-coherent transport to path lengths $L \leq l_{\phi}$, where the phase-decoherence mean free path $l_{\phi}$ typically allows only a moderate number of traversals across the cavity. The latter restriction rules out that very long paths with $L>l_{\phi}$ contribute to quantum interference in the experiment, a feature which is naturally incorporated by way of the cutoff length $L_{\max }$ in our semiclassical theory. Clearly, such long paths can still provide incoherent contributions. The present approach may thus contribute to a semiclassical understanding of decoherence effects in regular cavities. $^{28}$

In the present treatment of diffractive scattering, both internal diffraction at the open lead mouth giving rise to pseudopaths as well as the coupling between leads and cavity was performed for sharp edges. The weight of diffractive contributions can be changed by "rounding off" the lead opening. An investigation of the dependence of the weak localization on the smoothness of the edges is currently underway. ${ }^{61}$ The introduction of rounded corners has, however, another profound effect, apart from changing the weight of diffractive scattering: an open circle with rounded edges of the leads is no longer regular but features a mixed phase space. This raises the question as to the interplay between diffractive scatterings at the lead opening and chaotic scattering in the interior of the billiard. For generic chaotic systems a number of alternative semiclassical theories has been proposed to explain universal features of quantum transport (see, e.g., Refs. 17-23, and references therein). These theories typically employ an ensemble average and rely on a $\hbar \rightarrow 0$ limit which makes them complementary to the present system-specific approach for finite $\hbar$. Bridging the gap between these two frameworks would be of great interest. One key ingredient would be to clarify the interplay between the diffraction-based pseudopaths and the chaosbased correlated classical path pairs (Richter-Sieber orbits ${ }^{20}$ ). The relative weight for characteristic quantum-transport effects carried by these two classes of paths when both are present (as in a chaotic billiard with sharp edges) remains an open question.

\section{ACKNOWLEDGMENTS}

We thank Piet Brouwer, Tobias Dollinger, and Klaus Richter for helpful discussions. This work was supported by the Austrian FWF under Grants No. FWF-SFB016 "ADLIS" and No. 17359 and the FWF program "CoQuS." L.W. acknowledges support by the PHC "Amadeus" of the French Ministry of Foreign and European Affairs.

\section{APPENDIX: GEOMETRIC AND UNIFORM THEORY OF DIFFRACTION FOR THE COUPLING OF QUANTUM LEADS TO A BILLIARD CAVITY}

In a quantum billiard, the electron propagation in the leads is determined by a quantum-mechanical wave while the propagation inside the ballistic cavity can be described semiclassically, i.e., by propagation along classical trajectories with a quantum phase. The strong scattering effects that occur especially in the low mode regime at the orifices have been described in past works on transport through open billiards by the Kirchhoff diffraction approximation (KDA) (Ref. 10) or on the level of the FDA. ${ }^{12-15}$ In the high mode regime diffraction has been neglected altogether. ${ }^{8,17-24,26}$ Both the KDA and the FDA perform about equally well (see, e.g., Fig. 5). For the identification of pseudopaths in the Fourier spectra of the conductance fluctuations, the KDA and the FDA have been sufficiently precise. However, in order to recover unitarity of the semiclassical $S$-matrix and weak localization in transmission, a more precise diffraction theory must be implemented. For multiple-scattering paths, higherorder products of the diffraction weights occur and even small errors are rapidly amplified. We have therefore implemented a combination of the GTD (Ref. 32) and the UTD (Refs. 30 and 31) for the diffraction coefficients in open billiards referred to in the following as the GTD-UTD. Both theories have been previously applied separately to the calculation of higher-order scattering corrections to Gutzwiller's trace formula in closed quantum billiards. ${ }^{31}$ We discuss in Section 1 of Appendix diffractive backscattering at a semiinfinite half plane with an orifice and in Section 2 of Appendix diffraction during propagation from a lead into a semiinfinite plane.

\section{GTD-UTD for backscattering into the cavity}

The propagation between two points in a semi-infinite plane with a connected lead is depicted in Fig. 15. In the absence of a lead, the propagation between two points $\vec{r}$ and $\vec{r}^{\prime}$ in a semi-infinite plane is described by a sum of two Green's functions, $G\left(\vec{r}^{\prime}, \vec{r}, k\right)=G^{\operatorname{dir}}\left(\vec{r}^{\prime}, \vec{r}, k\right)+G^{\text {refl }}\left(\vec{r}^{\prime}, \vec{r}, k\right)$. The first contribution corresponds to the direct path from $\vec{r}$ to 


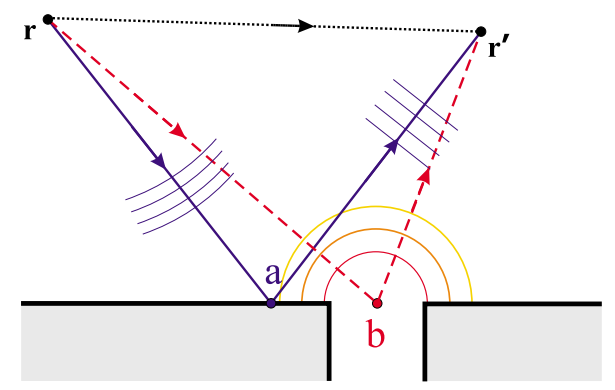

FIG. 15. (Color online) Propagation between two points $\vec{r}$ and $\vec{r}^{\prime}$ in a semi-infinite plane in the presence of an open (lead). Contributions from the direct path (black dotted line), from specular reflection (blue solid line), and from scattering at the orifice (red dashed line) are depicted.

$\vec{r}^{\prime}$ (black dotted line) and is in the semiclassical approximation [Eq. (12)] given by

$$
G^{\operatorname{dir}}\left(\vec{r}^{\prime}, \vec{r}, k\right)=\frac{e^{i k L\left(\vec{r}^{\prime}, \vec{r}\right)-i 3 \pi / 4-i \mu \pi / 2}}{\sqrt{2 \pi k L\left(\vec{r}^{\prime}, \vec{r}\right)}}=: G^{\mathrm{SCA}}\left(\vec{r}^{\prime}, \vec{r}, k\right),
$$

where $L\left(\vec{r}^{\prime}, \vec{r}\right)$ is the distance between the two points and $\mu$ is the Maslov index ( $\mu=0$ in this case). The second term corresponds to a propagation via a classical, specularly reflected path (blue solid line),

$$
G^{\mathrm{refl}}\left(\vec{r}^{\prime}, \vec{r}, k\right)=\frac{e^{i k\left[L\left(\vec{r}^{\prime}, \vec{a}\right)+L(\vec{a}, \vec{r})\right]-i 3 \pi / 4-i \pi}}{\sqrt{2 \pi k\left[L\left(\vec{r}^{\prime}, \vec{a}\right)+L(\vec{a}, \vec{r})\right]}} .
$$

The orifice gives rise to an additional scattered wave for which we assume in the far-field limit $\left(|\vec{k} \vec{r}| \gg 1\right.$ and $\left|\vec{k} \vec{r}^{\prime}\right|$ $\gg 1$ ) a cylindrical wave emanating from the center of the orifice (point $\vec{b}$ ). Invoking far-field approximations is at the heart of semiclassical diffraction theories, the validity of which need testing on a case-by-case basis (see below).

The diffraction contribution to the Green's function (red dashed line in Fig. 15) is

$$
\begin{aligned}
G^{\mathrm{PSCA}}\left(\vec{r}^{\prime}, \vec{r}, k\right) & =\frac{e^{i k L\left(\vec{r}^{\prime}, \vec{b}\right)-i 3 \pi / 4}}{\sqrt{2 \pi k L\left(\vec{r}^{\prime}, \vec{b}\right)}} v\left(\theta^{\prime}, \theta, k, d\right) \frac{e^{i k L(\vec{b}, \vec{r})-i 3 \pi / 4}}{\sqrt{2 \pi k L(\vec{b}, \vec{r})}} \\
& =G^{\mathrm{SCA}}\left(\vec{r}^{\prime}, \vec{b}, k\right) v\left(\theta^{\prime}, \theta, k, d\right) G^{\mathrm{SCA}}(\vec{b}, \vec{r}, k) .
\end{aligned}
$$

We refer to this term as the (first-order) pseudopath semiclassical contribution because it corresponds to a classically forbidden path. We note the different scaling of $G^{\mathrm{PSCA}}$ and $G^{\text {refl }}$ with $k$. The amplitude of $G^{\text {refl }}$ scales as $1 / \sqrt{k\left(L_{1}+L_{2}\right)}$ while the diffractive contribution scales as $1 / \sqrt{k^{2} L_{1} L_{2}}$. At large distances (far-field) and/or large $k$, the geometric reflection amplitude dominates over the diffraction amplitude, as expected.

The diffraction coefficient $v\left(\theta^{\prime}, \theta, k, d\right)$ as a function of the incoming and outgoing angles has been calculated in the past in the KDA and FDA. Both approaches have in common that the amplitude of the cylindrical diffractive wave emanating from the orifice is determined from an integration over
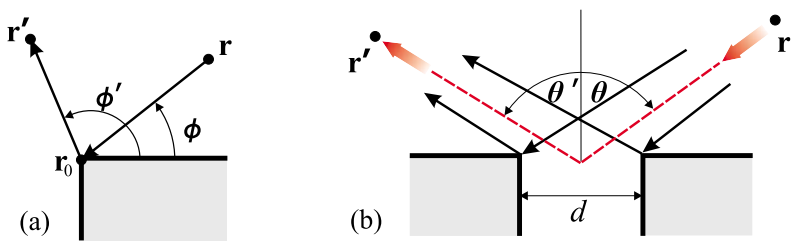

(c)

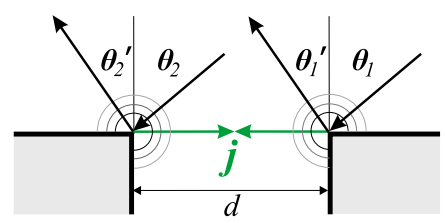

FIG. 16. (Color online) (a) Diffraction at a wedge. (b) Diffraction at a lead described as diffraction at two wedges. The reference path is marked by a red dashed line. The angle is counted positive for a path lying on the left of the vertical axis, for a path on the right the angle is negative. (In the present example $\theta$ is negative and $\theta^{\prime}$ is positive). The points $\vec{r}$ and $\vec{r}^{\prime}$ are assumed to be in the far-field region. (c) Higher-order corrections enter via paths scattered between the wedges.

the orifice using as a source the amplitude and the phase of the unperturbed incoming wave in the absence of the boundary. In other words, they are implementations of Huygens' principle according to which each point of the lead opening is the source of an outgoing circular wave. For details, we refer the reader to Ref. 10 (for the KDA) and to Refs. 12 and 14 (for the FDA).

Keller's GTD (Ref. 32) has a different point of departure: it originates from the far-field approximation of the exact Sommerfeld's solution for the wave scattering at a wedge ${ }^{62}$ [see Fig. 16(a)]. In the following, we discuss the application of the GTD and its refinement within the framework of the uniform theory of diffraction UTD (Ref. 30) to the problem of the diffractive scattering at the lead mouth [Figs. 16(b) and $16(\mathrm{c})]$. Starting point of our determination of the diffraction coefficient $v\left(\theta^{\prime}, \theta, k, d\right)$ is the decomposition of the orifice into two wedges with an inner angle of $\pi / 2$ and outer angle of $3 \pi / 2$ [see Fig. 16(a)]. In the far-field limit, the incident wave can be regarded asymptotically as a plane wave. The GTD describes the scattering of a plane wave at an infinitely sharp wedge ${ }^{32}$ [Fig. 16(a)]. The total wave function is a sum of the incoming plane wave, the reflected plane wave and an outgoing cylindrical diffracted wave, emanating from the edge. There are two discontinuities: at the shadow boundary $\left[\phi^{\prime}-\phi=\pi\right.$, for the definition of the angles see Fig. $16(\mathrm{a})]$ and the boundary of geometric reflection $\left(\phi^{\prime}+\phi=\pi\right)$. Outside a small region around these two angles, the diffracted wave can be described by an outgoing cylindrical wave modulated by a smooth diffraction coefficient. The diffractive part of the Green's function between the two points $\vec{r}$ and $\vec{r}^{\prime}$ in Fig. 16(a) can thus be approximated as ${ }^{63}$

$$
G^{\mathrm{PSCA}}\left(\vec{r}^{\prime}, \vec{r}, k\right)=G^{\mathrm{SCA}}\left(\vec{r}^{\prime}, \vec{r}_{0}, k\right) \frac{1}{2} D\left(\phi^{\prime}, \phi\right), G^{\mathrm{SCA}}\left(\vec{r}_{0}, \vec{r}, k\right)
$$

with 


$$
\begin{aligned}
D\left(\phi^{\prime}, \phi\right)= & -2 \frac{\sin \pi / N}{N}\left[\frac{1}{\cos \frac{\pi}{N}-\cos \frac{\phi^{\prime}-\phi}{N}}\right. \\
& \left.-\frac{1}{\cos \frac{\pi}{N}-\cos \frac{\phi^{\prime}+\phi}{N}}\right],
\end{aligned}
$$

where $N=3 / 2$ is the exterior angle (in units of $\pi$ ) of a perpendicular wedge and $\vec{r}_{0}$ is the position of the corner of the wedge [Fig. 16(a)].

We now consider the lead opening as being composed of two wedges [Fig. 16(b)]. The obvious conceptual difficulty lies in the fact that the two wedges are, in general, not in the far-field limit $(k d \gg 1)$ of each other. With this caveat in mind, diffraction at the lead can be considered within the GTD to result from the interference of two paths that are diffracted at the corners of the two wedges limiting the lead. Summing up the diffraction weights and phases from the two paths, we obtain the Green's functions of the reference pseudopath as in Eq. (A3) but with the GTD reflection coefficient

$$
\begin{aligned}
v^{\mathrm{GTD}}\left(\theta^{\prime}, \theta, k, d\right)= & \frac{1}{2} D_{L}\left(\theta^{\prime}, \theta\right) e^{-i k \frac{d}{2}\left(\sin \theta^{\prime}+\sin \theta\right)} \\
& +\frac{1}{2} D_{R}\left(\theta^{\prime}, \theta\right) e^{+i k \frac{d}{2}\left(\sin \theta^{\prime}+\sin \theta\right)}
\end{aligned}
$$

In Eq. (A6) we have neglected the difference of the incoming angles at the left and at the right wedge, respectively, i.e., we set $\theta_{1}=\theta_{2}=\theta$ [Fig. 16(c)]. Likewise, for the outgoing angles, we set $\theta_{1}^{\prime}=\theta_{2}^{\prime}=\theta^{\prime}$. The phase differences of the right/left pseudopath with respect to the reference path emanating from the center of the orifice can then be written in linear approximation with respect to the transverse lead coordinate as $k \Delta L= \pm i k \frac{d}{2}\left(\sin \theta^{\prime}+\sin \theta\right)$. The coefficients in Eq. (A6) are defined as

$$
\begin{aligned}
& D_{L}\left(\theta^{\prime}, \theta\right)=D\left(\pi / 2-\theta^{\prime}, \pi / 2-\theta\right), \\
& D_{R}\left(\theta^{\prime}, \theta\right)=D\left(\pi / 2+\theta^{\prime}, \pi / 2+\theta\right) .
\end{aligned}
$$

We note that both $D_{L}$ and $D_{R}$ have a singularity at the reflection boundary for $\theta^{\prime}=-\theta$. However, in the sum of the two terms, the singularities cancel out and the resulting backscattering amplitude is perfectly smooth (see Fig. 17, red chain dotted line).

The comparison of the GTD with the exact quantum calculation for the diffraction coefficient, $\left|v\left(\theta, \theta^{\prime}, k, d\right)\right|^{2}$ (Fig. 17), reveals sizeable deviations. Two deficiencies are noteworthy: the almost complete missing of the back-reflection peak and the failure at grazing angles $\theta^{\prime}, \theta \rightarrow \neq \pi / 2$. The diffraction coefficient should approach zero in this limit but, instead, converges toward a finite value (Fig. 17). This behavior originates from treating the diffraction at the lead as two independent local phenomena of diffraction at two separate wedges.

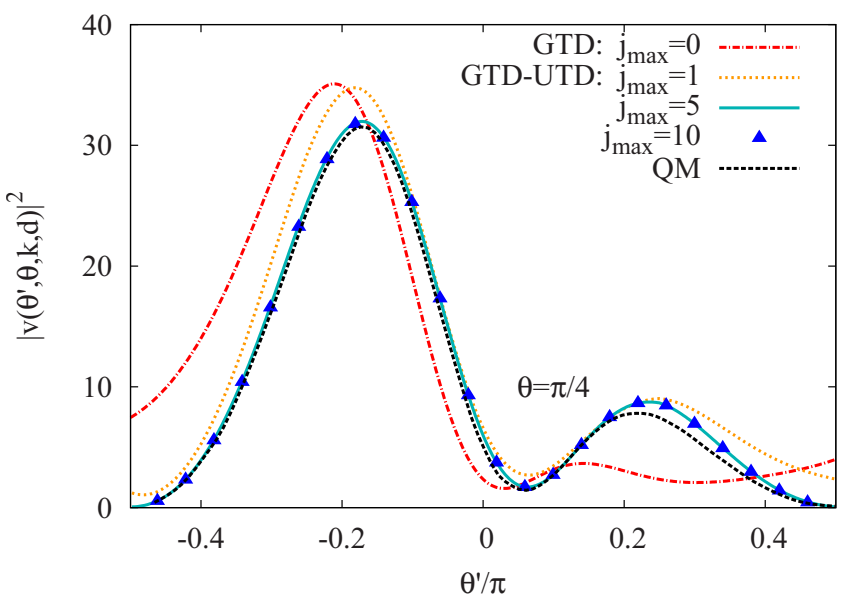

FIG. 17. (Color online) Absolute square of the diffraction coefficient $\left|v^{\mathrm{GTD}}\left(\theta^{\prime}, \theta, k, d\right)\right|^{2}$ (or $v^{\mathrm{GTD}-\mathrm{UTD}}$ with $j_{\max }=0$ ) compared with $\left|v^{\mathrm{GTD}-\mathrm{UTD}}\left(\theta^{\prime}, \theta, k, d\right)\right|^{2}$ for $j_{\max }=1, j_{\max }=5$, and $j_{\max }=10$ (for the definition of $j_{\text {max }}$ see text), $k=2.5 \pi / d$. The QM result is taken from Ref. 10.

The diffraction at a lead can be treated within the UTD by using a double-wedge diffraction coefficient (see Refs. 64 and 65 and a more recent paper for arbitrary configurations of the wedges, Ref. 66). The double-wedge diffraction coefficient cannot be separated into a sequence of single-wedge diffraction coefficients and contains rather involved mathematical expressions (such as two-dimensional Fresnel integrals) such that the beauty and structural simplicity of a semiclassical approach is lost. We present in the following an ansatz for double-wedge diffraction which assumes a separation of the diffraction process into a sequence of diffraction events and we verify its validity by comparing with quantum-mechanical results from Ref. 10. We show that the shortcomings of Eq. (A6) can be (to a large extent) remedied by taking diffractive paths of higher order into account, i.e., paths that pass between the two wedges once or several times. This drastically improves the agreement with the quantum-mechanical result. (We point to the conceptual similarity of our approach to the treatment of double-wedge diffraction illuminated by transition region fields by a sum over higher-order diffracted fields. ${ }^{67}$ )

In a first step, we include paths that scatter once between the two edges [see green line Fig. 16(c)]. There are two such paths. One approaches the right wedge at angle $\theta$. It is scattered into the angle $\pi / 2$ (with respect to the surface normal), and at the left wedge, it is scattered into the angle $\theta^{\prime}$. The other path is scattered from the left wedge to the right one with the same entrance and exit angles. The weights of this pair of paths cannot be determined from the GTD diffraction coefficients [Eq. (A5)]: The GTD diffraction coefficient fails in the limit of $\phi \rightarrow 0$ and $\phi^{\prime} \rightarrow \pi$ [definition of angles as in Fig. 16(a)] because this is in proximity of the shadow boundary into which the horizontal paths are scattered. This problem can be overcome by invoking the UTD.

Contrary to the GTD, the UTD is also valid on the zone boundaries. The outgoing cylindrical wave is multiplied by a diffraction coefficient which depends not only on the two angles $\phi^{\prime}$ and $\phi$ but also on the distances $r^{\prime}$ and $r$, and on the wave number $k$, 


$$
\begin{aligned}
D^{\mathrm{UTD}}\left(\phi^{\prime}, \phi, r^{\prime}, r, k\right)= & -\frac{e^{i \pi / 4}}{N} \sum_{\sigma, \eta= \pm 1} \sigma \cot \left[\frac{\pi+\eta\left(\phi^{\prime}-\sigma \phi\right)}{2 N}\right] \\
& \times F\left[k \frac{r r^{\prime}}{r+r^{\prime}} a_{\eta}\left(\phi^{\prime}-\sigma \phi\right)\right],
\end{aligned}
$$

where $a_{ \pm}(\beta)=2 \cos ^{2}\left(\frac{2 \pi N n^{ \pm}-\beta}{2}\right)$ and $n^{ \pm}$is the integer which most closely satisfies $2 \pi N n^{ \pm}-\beta= \pm \pi$. The function $F$ is defined as a generalized Fresnel integral,

$$
F(x)=-2 i \sqrt{x} e^{-i x} \int_{\sqrt{x}}^{\infty} d \tau e^{i \tau^{2}}
$$

and has the asymptotic form

$$
F(x)=1-i \frac{1}{2 x}-\frac{3}{4} \frac{1}{x^{2}}+\cdots .
$$

For $x \rightarrow \infty$, i.e., for large distances and outside the transition zones, $\quad F(x)=1$, and $D^{\mathrm{UTD}}\left(\phi^{\prime}, \phi, r^{\prime}, r, k\right)$ reduces to $D^{\mathrm{GTD}}\left(\phi^{\prime}, \phi\right)$. Within the transition zone, the distance dependence of $D^{\text {UTD }}$ leads to a deviation of the scattered wave from a purely cylindrical wave. This is necessary to ensure the continuity of the total wave function at the zone boundaries. Furthermore, by using the UTD diffraction coefficient we partially take into account the fact that the two wedges are not in the far-field region with respect to each other.

Since the GTD fails for large (near grazing) angles, we opt for a piecewise construction. We combine GTD and UTD referred to in the following as GTD-UTD such that the diffraction of the path with the smaller (absolute value) of the angle is treated by the GTD and the path with the larger angle on the level of the UTD. Accordingly, to first order the GTD-UTD correction to the diffraction coefficient can be written as

$$
\begin{gathered}
v^{\text {GTD-UTD }, 1}\left(\theta^{\prime}, \theta, k, d\right)=\frac{1}{2} U_{L}\left(\theta^{\prime},-\pi / 2, d, k\right) \\
\times \frac{e^{i k d\left(-\sin \theta^{\prime}+\sin \theta\right) / 2}}{\sqrt{2 \pi k d}} \frac{1}{2} D_{R}(+\pi / 2, \theta) \\
+\frac{1}{2} U_{R}\left(\theta^{\prime},+\pi / 2, d, k\right) \\
\times \frac{e^{i k d\left(+\sin \theta^{\prime}-\sin \theta\right) / 2}}{\sqrt{2 \pi k d}} \frac{1}{2} D_{L}(-\pi / 2, \theta),
\end{gathered}
$$

where [in analogy to Eq. (A7)] we have defined the UTD diffraction coefficients at the left $(L)$ and right $(R)$ wedge as

$$
\begin{aligned}
& U_{L}\left(\theta^{\prime}, \theta, r, k\right)=D^{\mathrm{UTD}}\left(\pi / 2-\theta^{\prime}, \pi / 2-\theta, r^{\prime} \rightarrow \infty, r, k\right), \\
& U_{R}\left(\theta^{\prime}, \theta, r, k\right)=D^{\mathrm{UTD}}\left(\pi / 2+\theta^{\prime}, \pi / 2+\theta, r^{\prime} \rightarrow \infty, r, k\right) .
\end{aligned}
$$

In Eq. (A11), the diffraction of the incoming path (with angle $\theta$ ), is treated on the GTD level and the diffraction of the outgoing path (angle $\theta^{\prime}$ ) on the UTD level. This construction

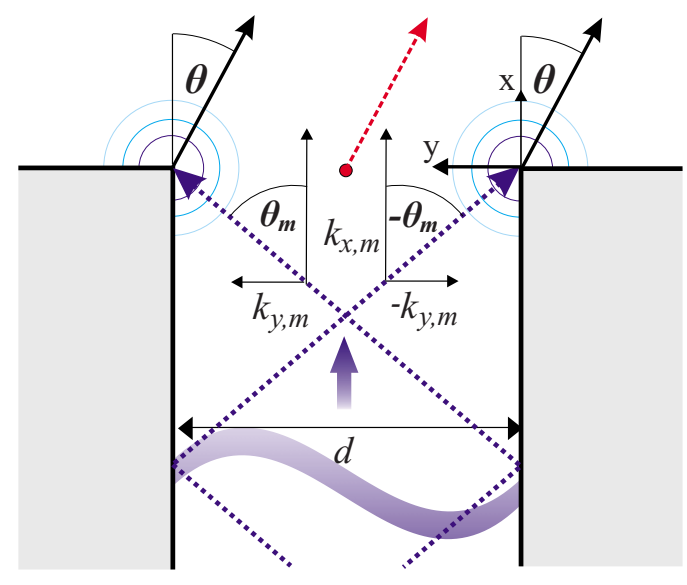

FIG. 18. (Color online) A lead of width $d$ coupled to a halfinfinite plane. An incoming wave in mode $m$ can be separated into two rays $\theta= \pm \theta_{m}= \pm \arcsin m \pi / d k$ which diffractively scatter at the lead wedges. The reference path is denoted by a red dashed line.

introduces a first-order discontinuity (kink) at $|\theta|=\left|\theta^{\prime}\right|$. This kink is, however, negligible for small angles and visible only at large angles close to $\pi / 2$, in other words the formula breaks down in the limit $\theta, \theta^{\prime} \rightarrow \pi / 2$, see, e.g., Fig. 5(c). (One could alternatively ignore the fact that the UTD is not multiplicative in the near field and employ for both scattering events UTD, thus avoiding the kink. This ansatz, however, breaks down similarly for grazing incidence: the diffraction coefficient does not approach zero for the outgoing angle $\theta^{\prime} \rightarrow \pi / 2$. For small and medium angles this approach behaves equally well as the presented GTD-UTD approach.) At large incident and/or outgoing angles the diffraction coefficient is already strongly suppressed such that the resulting error is small.

The inclusion of the first-order GTD-UTD correction $\left(j_{\max }=1\right.$, see Fig. 17) already considerably improves the agreement with the quantum diffraction pattern. Higher-order diffraction corrections include paths that are scattered several times between the wedges. This includes paths that are incident and backscattered at an angle $\pm \pi / 2$ at the wedge. This is exactly on the reflection boundary. In this limit, Reiche has shown ${ }^{68}$ that the diffraction pattern of a plane wave with unit amplitude incident on the wedge with an angle of $\pm \pi / 2$ reduces to a reflected plane wave with amplitude 1/2 and a cylindrical wave. The Green's function of a higher-order path which scatters $j$ times between the wedges is, therefore, a product of the GTD diffraction coefficient, the UTD diffraction coefficient, and the Green's function for free propagation along the distance $j d$, acquiring a factor $1 / 2$ and a phase of $\pi$ for each reflection at a wedge. Summing the diffraction corrections up to order $j_{\max }$ we obtain

$$
\begin{aligned}
& v^{\mathrm{GTD}-\mathrm{UTD}}\left(\theta^{\prime}, \theta, k, d\right)=v^{\mathrm{GTD}}\left(\theta^{\prime}, \theta, k, d\right) \\
& +\frac{1}{4} \sum_{\text {odd }: j=1}^{j_{\max }} U_{L}\left(\theta^{\prime},-\pi / 2, j d, k\right) g_{j}(k) e^{i \Phi_{-+}} D_{R}(+\pi / 2, \theta) \\
& +U_{R}\left(\theta^{\prime},+\pi / 2, j d, k\right) g_{j}(k) e^{i \Phi_{+-} D_{L}(-\pi / 2, \theta)}
\end{aligned}
$$



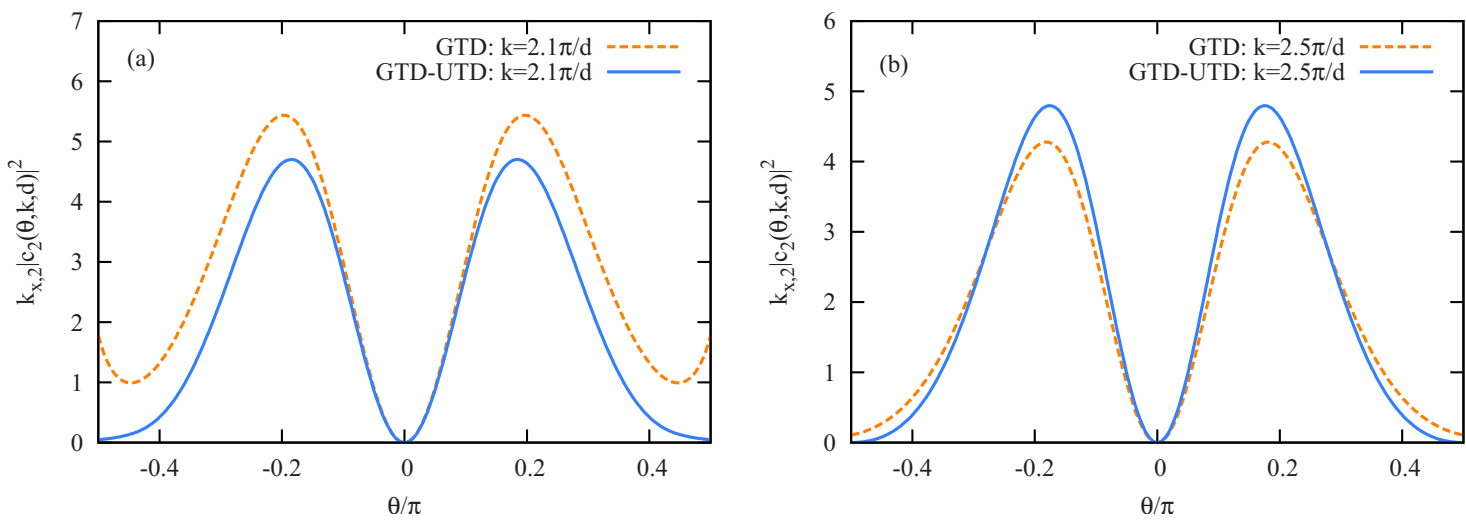

FIG. 19. (Color online) The absolute square of the coupling coefficient $\left|c_{m}(\theta, k, d)\right|^{2}$ multiplied by $k_{x, m}$ for $m=2$ at two different wave numbers (a) $k=2.1 \pi / d$ and (b) $k=2.5 \pi / d$ within the GTD and the GTD-UTD. Note the breakdown of the GTD for $\theta \rightarrow \pm \pi / 2$ near $k$ $\approx 2 \pi / d$.

$$
\begin{aligned}
& +\frac{1}{4} \sum_{\text {even:j=1 }}^{j_{\max }} U_{R}\left(\theta^{\prime},+\pi / 2, j d, k\right) g_{j}(k) e^{i \Phi_{++}} D_{R}(+\pi / 2, \theta) \\
& +U_{L}\left(\theta^{\prime},-\pi / 2, j d, k\right) g_{j}(k) e^{i \Phi_{--}} D_{L}(-\pi / 2, \theta),
\end{aligned}
$$

where

$$
g_{j}(k)=\frac{1}{\sqrt{2 \pi k j d}} \frac{1}{2^{j-1}} e^{i[k j d+(j-1) \pi]}
$$

and

$$
\Phi_{ \pm \pm}=k \frac{d}{2}\left( \pm \sin \theta^{\prime} \pm \sin \theta\right) .
$$

Figure 17 demonstrates that the diffraction coefficient $v\left(\theta^{\prime}, \theta, k, d\right)^{\text {GTD-UTD }}$ is converged for $j_{\text {max }}=5$. Furthermore, the condition $v\left(\theta^{\prime}, \theta, k, d\right) \rightarrow 0$ for $\theta, \theta^{\prime} \rightarrow \pi / 2$ is fulfilled and the agreement with the fully quantum-mechanical backscattering weight is excellent. The agreement deteriorates for increasing entrance angles $\theta$ but is still satisfying compared to the simple FDA, (see Fig. 5). Fortunately, large angles do not play an important role because the overall diffraction weight is very low. The GTD-UTD provides a remarkable compromise between simplicity and accurate representation of quantum-mechanical results for diffraction at a lead attached to a semi-infinite half plane.

\section{GTD-UTD for coupling from lead modes into the cavity}

The flux-normalized wave function for mode $m$ in a lead of width $d$ oriented parallel to the $x$ axis is

$$
\begin{aligned}
\psi_{m}(x, y) & =\sqrt{\frac{2}{d k_{x, m}} e^{i k_{x, m} x} \sin \left(k_{y, m} y\right)} \\
& =-i \sqrt{\frac{1}{2 d k_{x, m}}}\left[e^{i\left(k_{x, m} x+k_{y, m} y\right)}-e^{i\left(k_{x, m} x-k_{y, m} y\right)}\right],
\end{aligned}
$$

where $k_{y, m}=m \pi / d$ is the transverse momentum component and $k_{x, m}=\sqrt{k^{2}-(m \pi / d)^{2}}$ the longitudinal component. Equa- tion (A16) can be viewed as two rays emanating with angles $\pm \theta_{m}= \pm \arcsin (m \pi / d k)$ (Fig. 18). In the GTD approximation, each ray hits an edge of the lead mouth and the two cylindrical waves emanating from the edges cause, in turn, an interference pattern at large distances. The GTD diffraction for scattering from the lead mode $m$ into the half space (in our application the cavity) can be written in direct analogy with Eqs. (A6) and (A7) as

$$
\begin{aligned}
c_{m}^{\mathrm{GTD}}(\theta, k, d)= & \frac{-i e^{i m \pi / 2}}{\sqrt{2 d} k_{x, m}}\left[\frac{1}{2} D_{L}\left(\theta, \theta_{m}\right) e^{i m \pi / 2} e^{-i k d / 2 \sin \theta}\right. \\
& \left.-\frac{1}{2} D_{R}\left(\theta, \theta_{m}\right) e^{-i m \pi / 2} e^{i k d / 2 \sin \theta}\right]
\end{aligned}
$$

with the scattering coefficients at left and right wedges,

$$
D_{L}\left(\theta, \theta_{m}\right)=D\left(\frac{\pi}{2}-\theta, \frac{3 \pi}{2}-\theta_{m}\right) \text {, }
$$

The two paths have a phase difference of $m \pi$ at the lead mouth and thus a phase difference $\pm m \pi / 2$ relative to the reference path that starts at the center of the orifice (see Fig. 18). With the flux normalization factor $\sqrt{k_{x, m}}$ from Eq. (2) $\sqrt{k_{x, m}} c_{m}^{\mathrm{GTD}}(\theta, k, d)$ is dimensionless.

We include now higher-order scattering events on the level of the UTD in order to improve Eq. (A17), 


$$
\begin{aligned}
& c_{m}^{\mathrm{GTD}-\mathrm{UTD}}(\theta, k, d)=c_{m}^{\mathrm{GTD}}(\theta, k, d)-i \frac{e^{i m \pi / 2}}{\sqrt{2 d} k_{x, m}} \frac{1}{4}\left[\sum_{\text {odd }: j=1}^{j_{\max }} U_{R}(\theta,+\pi / 2, j d, k) g_{j}(k) e^{i \phi_{++}} D_{L}\left(-\pi / 2, \theta_{m}\right)-U_{L}(\theta,-\pi / 2, j d, k) g_{j}(k)\right.
\end{aligned}
$$

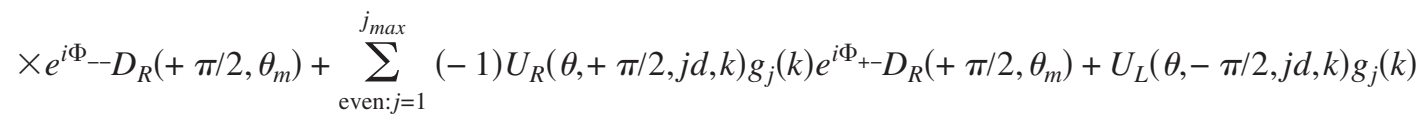

$$
\begin{aligned}
& \times e^{\left.i \Phi_{-+} D_{L}\left(-\pi / 2, \theta_{m}\right)\right]}
\end{aligned}
$$

where

$$
\Phi_{ \pm \pm}= \pm k \frac{d}{2} \sin \theta \pm \frac{m \pi}{2},
$$

$g_{j}(k)$ is given in Eq. (A14), and $U_{L}$ and $U_{R}$ are given by Eq. (A12). Figure 19 illustrates that the UTD corrections become most important when the value of $k$ is close to a threshold, i.e., when the angle $\theta_{m}$ is close to $\pi / 2$.

*iva.brezinova@tuwien.ac.at

${ }^{1}$ S. Datta, Electronic Transport in Mesoscopic Systems (Cambridge University Press, Cambridge, England, 1995).

${ }^{2}$ E. Akkermans and G. Montambaux, Mesoscopic Physics of Electrons and Photons (Cambridge University Press, Cambridge, England, 2006).

${ }^{3}$ C. W. J. Beenakker and H. van Houten, Solid State Phys. 44, 1 (1991).

${ }^{4}$ M. C. Gutzwiller, Chaos in Classical and Quantum Mechanics (Springer-Verlag, New York, 1991).

${ }^{5}$ N. V. Berry and K. E. Mount, Rep. Prog. Phys. 35, 315 (1972).

${ }^{6}$ M. Brack and R. K. Bhaduri, Semiclassical Physics, Frontiers in Physics (Westview, Oxford, 2003).

${ }^{7}$ R. Blümel and U. Smilansky, Phys. Rev. Lett. 64, 241 (1990).

${ }^{8}$ H. U. Baranger, R. A. Jalabert, and A. D. Stone, Chaos 3, 665 (1993).

${ }^{9}$ T. Blomquist, Phys. Rev. B 66, 155316 (2002).

${ }^{10}$ C. D. Schwieters, J. A. Alford, and J. B. Delos, Phys. Rev. B 54, 10652 (1996).

${ }^{11}$ T. Blomquist and I. V. Zozoulenko, Phys. Rev. B 64, 195301 (2001).

${ }^{12}$ L. Wirtz, J.-Z. Tang, and J. Burgdörfer, Phys. Rev. B 56, 7589 (1997).

${ }^{13}$ L. Wirtz, J.-Z. Tang, and J. Burgdörfer, Phys. Rev. B 59, 2956 (1999).

${ }^{14}$ L. Wirtz, C. Stampfer, S. Rotter, and J. Burgdörfer, Phys. Rev. E 67, 016206 (2003).

${ }^{15}$ C. Stampfer, S. Rotter, J. Burgdörfer, and L. Wirtz, Phys. Rev. E 72, 036223 (2005).

${ }^{16}$ I. Březinová, C. Stampfer, L. Wirtz, S. Rotter, and J. Burgdörfer, Phys. Rev. B 77, 165321 (2008).

${ }^{17}$ S. Rahav and P. W. Brouwer, Phys. Rev. Lett. 95, 056806 (2005).

${ }^{18}$ S. Rahav and P. W. Brouwer, Phys. Rev. B 73, 035324 (2006).

${ }^{19}$ P. Jacquod and R. S. Whitney, Phys. Rev. B 73, 195115 (2006).

${ }^{20}$ K. Richter and M. Sieber, Phys. Rev. Lett. 89, 206801 (2002).

${ }^{21}$ P. Braun, S. Heusler, S. Müller, and F. Haake, J. Phys. A 39, L159 (2006).
${ }^{22}$ S. Heusler, S. Müller, P. Braun, and F. Haake, Phys. Rev. Lett. 96, 066804 (2006).

${ }^{23}$ P. W. Brouwer and S. Rahav, Phys. Rev. B 74, 085313 (2006).

${ }^{24}$ P. Pichaureau and R. A. Jalabert, Eur. Phys. J. B 9, 299 (1999).

${ }^{25}$ E. Bogomolny, Nonlinearity 13, 947 (2000).

${ }^{26}$ N. Argaman, Phys. Rev. B 53, 7035 (1996).

${ }^{27}$ R. P. Feynman and A. R. Hibbs, Quantum Mechanics and Path Integrals (McGraw-Hill, New York, 1965).

${ }^{28}$ A. M. Chang, H. U. Baranger, L. N. Pfeiffer, and K. W. West, Phys. Rev. Lett. 73, 2111 (1994).

${ }^{29}$ C. M. Marcus, A. J. Rimberg, R. M. Westervelt, P. F. Hopkins, and A. C. Gossard, Phys. Rev. Lett. 69, 506 (1992).

${ }^{30}$ R. Kouyoumjian and P. H. Pathak, Proc. IEEE 62, 1448 (1974).

${ }^{31}$ M. Sieber, N. Pavloff, and C. Schmit, Phys. Rev. E 55, 2279 (1997).

${ }^{32}$ J. B. Keller, J. Opt. Soc. Am. 52, 116 (1962).

${ }^{33}$ R. Brunner, R. Meisels, F. Kuchar, R. Akis, D. K. Ferry, and J. P. Bird, Phys. Rev. Lett. 98, 204101 (2007).

${ }^{34}$ S. Rotter, J.-Z. Tang, L. Wirtz, J. Trost, and J. Burgdörfer, Phys. Rev. B 62, 1950 (2000).

${ }^{35}$ S. Rotter, B. Weingartner, N. Rohringer, and J. Burgdörfer, Phys. Rev. B 68, 165302 (2003).

${ }^{36}$ H. Ishio and J. Burgdörfer, Phys. Rev. B 51, 2013 (1995).

${ }^{37}$ D. S. Fisher and P. A. Lee, Phys. Rev. B 23, 6851 (1981).

${ }^{38}$ T. Szeredi and D. A. Goodings, Phys. Rev. E 48, 3529 (1993).

${ }^{39}$ G. Vattay, A. Wirzba, and P. E. Rosenqvist, Phys. Rev. Lett. 73, 2304 (1994).

${ }^{40}$ N. Pavloff and C. Schmit, Phys. Rev. Lett. 75, 61 (1995).

${ }^{41}$ S. Hikami, Phys. Rev. B 24, 2671 (1981).

${ }^{42}$ A. Andreev, Sov. Phys. JETP 19, 1228 (1964).

${ }^{43}$ X. Yang, H. Ishio, and J. Burgdörfer, Phys. Rev. B 52, 8219 (1995).

${ }^{44}$ J. Tworzydło, A. Tajic, and C. W. J. Beenakker, Phys. Rev. B 69, 165318 (2004).

${ }^{45}$ P. Jacquod and E. V. Sukhorukov, Phys. Rev. Lett. 92, 116801 (2004).

${ }^{46}$ M. Y. Kharitonov and K. B. Efetov, Phys. Rev. B 78, 033404 (2008). 
${ }^{47}$ B. Weingartner, S. Rotter, and J. Burgdörfer, Phys. Rev. B 72, 115342 (2005).

${ }^{48}$ M. Hartung, T. Wellens, C. A. Müller, K. Richter, and P. Schlagheck, Phys. Rev. Lett. 101, 020603 (2008).

${ }^{49}$ E. Larose, L. Margerin, B. A. van Tiggelen, and M. Campillo, Phys. Rev. Lett. 93, 048501 (2004).

${ }^{50}$ J. P. Robinson, H. Schomerus, L. Oroszlány, and V. I. Fal'ko, Phys. Rev. Lett. 101, 196803 (2008).

${ }^{51}$ M. Kopp, H. Schomerus, and S. Rotter, Phys. Rev. B 78, 075312 (2008).

${ }^{52}$ V. A. Gopar, S. Rotter, and H. Schomerus, Phys. Rev. B 73, 165308 (2006).

${ }^{53}$ Y. M. Blanter and M. Büttiker, Phys. Rep. 336, 1 (2000).

${ }^{54}$ F. Aigner, S. Rotter, and J. Burgdörfer, Phys. Rev. Lett. 94, 216801 (2005).

${ }^{55}$ S. Rotter, F. Aigner, and J. Burgdörfer, Phys. Rev. B 75, 125312 (2007).

${ }^{56}$ B. A. Khoruzhenko, D. V. Savin, and H.-J. Sommers, Phys. Rev. B 80, 125301 (2009).

${ }^{57}$ M. Novaes, Phys. Rev. B 75, 073304 (2007).
${ }^{58}$ J. Tworzydło, A. Tajic, H. Schomerus, and C. W. J. Beenakker, Phys. Rev. B 68, 115313 (2003).

${ }^{59}$ C. H. Lewenkopf, E. R. Mucciolo, and A. H. Castro Neto, Phys. Rev. B 77, 081410(R) (2008).

${ }^{60}$ J. Tworzydło, B. Trauzettel, M. Titov, A. Rycerz, and C. W. J. Beenakker, Phys. Rev. Lett. 96, 246802 (2006).

${ }^{61} \mathrm{~T}$. Dollinger et al. (unpublished).

${ }^{62}$ A. Sommerfeld, Vorlesungen über Theoretische Physik, Optik (Dieterich'sche Verlagsbuchhandlung, Wiesbaden, 1950).

${ }^{63}$ The factor $1 / 2$ (atomic units) in Eq. (A4) originates from $\hbar^{2} / 2 m$ (SI units) and assures that the Green's function is correctly normalized [such that $\left(\frac{\hbar^{2} k^{2}}{2 m}-\hat{H}_{\vec{r}^{\prime}}\right) G\left(\vec{r}^{\prime}, \vec{r}, k\right)=\delta\left(\vec{r}^{\prime}-\vec{r}\right)$ ]. We use the same normalization as in Ref. 6, section 7.5.4.

${ }^{64}$ M. Schneider and R. J. Luebbers, IEEE Trans. Antennas Propag. 39, 8 (1991).

${ }^{65}$ M. Schneider, Ph.D. thesis, Pennsylvania State University, 1988.

${ }^{66}$ M. Albani, IEEE Trans. Antennas Propag. 53, 702 (2005).

${ }^{67}$ P. D. Holm, IEEE Trans. Antennas Propag. 44, 879 (1996).

${ }^{68}$ F. Reiche, Ann. Phys. 37, 131 (1911). 